\title{
The University of Washington Ice-Liquid Discriminator (UWILD) improves single-particle phase classifications of hydrometeors within Southern Ocean clouds using machine learning
}

\author{
Rachel Atlas $^{1}$, Johannes Mohrmann ${ }^{1}$, Joseph Finlon $^{1}$, Jeremy Lu $^{1, \star}$, Ian Hsiao $^{1, \star}$, Robert Wood ${ }^{1}$, and \\ Minghui Diao ${ }^{2}$ \\ ${ }^{1}$ Department of Atmospheric Sciences, University of Washington, Seattle, WA, USA \\ ${ }^{2}$ Department of Meteorology and Climate Science, San Jose State University, San Jose, CA, USA \\ * These authors contributed equally to this work.
}

Correspondence: Rachel Atlas (ratlas@uw.edu)

Received: 2 May 2021 - Discussion started: 21 May 2021

Revised: 14 August 2021 - Accepted: 28 August 2021 - Published: 11 November 2021

\begin{abstract}
Mixed-phase Southern Ocean clouds are challenging to simulate, and their representation in climate models is an important control on climate sensitivity. In particular, the amount of supercooled water and frozen mass that they contain in the present climate is a predictor of their planetary feedback in a warming climate. The recent Southern Ocean Clouds, Radiation, Aerosol Transport Experimental Study (SOCRATES) vastly increased the amount of in situ data available from mixed-phase Southern Ocean clouds useful for model evaluation. Bulk measurements distinguishing liquid and ice water content are not available from SOCRATES, so single-particle phase classifications from the Two-Dimensional Stereo (2D-S) probe are invaluable for quantifying mixed-phase cloud properties. Motivated by the presence of large biases in existing phase discrimination algorithms, we develop a novel technique for singleparticle phase classification of binary 2D-S images using a random forest algorithm, which we refer to as the University of Washington Ice-Liquid Discriminator (UWILD). UWILD uses 14 parameters computed from binary image data, as well as particle inter-arrival time, to predict phase. We use liquidonly and ice-dominated time periods within the SOCRATES dataset as training and testing data. This novel approach to model training avoids major pitfalls associated with using manually labeled data, including reduced model generalizability and high labor costs. We find that UWILD is well calibrated and has an overall accuracy of $95 \%$ compared to $72 \%$ and $79 \%$ for two existing phase classification algo-
\end{abstract}

rithms that we compare it with. UWILD improves classifications of small ice crystals and large liquid drops in particular and has more flexibility than the other algorithms to identify both liquid-dominated and ice-dominated regions within the SOCRATES dataset. UWILD misclassifies a small percentage of large liquid drops as ice. Such misclassified particles are typically associated with model confidence below $75 \%$ and can easily be filtered out of the dataset. UWILD phase classifications show that particles with area-equivalent diameter $\left(D_{\text {eq }}\right)<0.17 \mathrm{~mm}$ are mostly liquid at all temperatures sampled, down to $-40^{\circ} \mathrm{C}$. Larger particles $\left(D_{\text {eq }}>\right.$ $0.17 \mathrm{~mm}$ ) are predominantly frozen at all temperatures below $0{ }^{\circ} \mathrm{C}$. Between 0 and $5^{\circ} \mathrm{C}$, there are roughly equal numbers of frozen and liquid mid-sized particles $\left(0.17<D_{\text {eq }}<\right.$ $0.33 \mathrm{~mm})$, and larger particles $\left(D_{\mathrm{eq}}>0.33 \mathrm{~mm}\right)$ are mostly frozen. We also use UWILD's phase classifications to estimate sub- $1 \mathrm{~Hz}$ phase heterogeneity, and we show examples of meter-scale cloud phase heterogeneity in the SOCRATES dataset.

\section{Introduction}

\subsection{Southern Ocean cloud phase and climate}

Mixed-phase processes within Southern Ocean clouds moderate cloud radiative effects (Bodas-Salcedo et al., 2016; McCoy et al., 2014a) and cloud-climate feedbacks associated 
with the stormy region (McCoy et al., 2014b). The presence of small amounts of ice within liquid-dominated mixedphase clouds can substantially increase precipitation as compared with warm clouds with similar thickness, due to efficient cold precipitation formation (Bergeron, 1928; Field and Heymsfield, 2015). Increased precipitation can reduce cloud lifetime (Albrecht, 1989) and increase aerosol scavenging (Radke et al., 1980).

The distribution of liquid and frozen hydrometeors within Southern Ocean clouds will change as the climate warms (Mitchell et al., 1989; Storelvmo et al., 2015). Climate models that simulate a relatively high ice-to-liquid ratio within Southern Ocean clouds in the present climate, or base state, exhibit a negative cloud radiative feedback in future climates (McCoy et al., 2015). One explanation for this is that as the climate warms, fewer ice particles form, and clouds become both brighter (Sun and Shine, 1994) and longer lived. This feedback, known as the cloud phase feedback, has a large impact on the climate sensitivity, and its weakening has been suggested as an explanation for an increase in climate sensitivity going from CMIP5 (Coupled Model Intercomparison Project) to CMIP6 (Zelinka et al., 2020; Bjordal et al., 2020).

Given that the strength of the cloud phase feedback in climate models is related to the base state representation of cloud phase within Southern Ocean clouds, we can constrain the cloud phase feedback by assessing how realistic the base state of each climate model is. Historically, due to a lack of in situ measurements over the Southern Ocean, satellite data have been used to evaluate cloud phase within climate models. Tan et al. (2016) retrieved cloud phase from CloudAerosol Lidar with Orthogonal Polarization (CALIOP) to show that climate models with strong cloud phase feedbacks typically underestimate the fraction of supercooled water in present-day extra-tropical, mixed-phase clouds. McCoy et al. (2014a) combined retrievals from the International Satellite Cloud Climatology Project (ISCCP), Moderate Resolution Imaging Spectroradiometer (MODIS), and Multi-angle Imaging Spectroradiometer (MISR) to connect seasonally varying cloud radiative fluxes with mixed-phase cloud properties. Satellite products have been invaluable for constraining cloud radiative effects and identifying model biases over the Southern Ocean, but they provide almost no information on hydrometeor phase other than at the cloud top. For this reason and because of low vertical and horizontal resolution and large retrieval uncertainties, satellite products are not sufficient to support process-oriented studies of mixedphase microphysics in models.

Recent in situ measurements of summertime Southern Ocean clouds from the Southern Ocean Clouds, Radiation, Aerosol Transport Experimental Study (SOCRATES; McFarquhar et al., 2020) make it possible to quantify cloud microphysical properties through the full depth of the cloud and to create a dataset for evaluating models and remote sensing retrievals. Since measurements of bulk ice water content (IWC) are not available during SOCRATES, single- particle classification is the most viable way to quantify ice properties within SOCRATES-sampled clouds. Furthermore, single-particle phase classifications are useful for making direct comparisons of simulated and observed liquid and frozen particle size distributions (PSDs). During SOCRATES, optical array probes (OAPs) such as the Two-Dimensional Stereo (2D-S) instrument (Lawson et al., 2006) collected binary images of single particles that can be used for phase classification. However, there is no standard procedure for identifying the phase of 2D-S imaged particles, and existing algorithms, which are described in Sect. 1.2, have substantial biases. Here, we introduce a novel machine-learning-based algorithm, the University of Washington Ice-Liquid Discriminator (UWILD), and show that it has greater skill in discriminating liquid and ice particles than two pre-existing algorithms.

\subsection{Existing methods for phase classification}

Single-particle phase classification techniques for binary OAP images typically distinguish liquid from frozen hydrometeors using particle shape and/or particle roughness. Cober et al. (2001a) used four different ratios computed from particle length, width, perimeter, and area to estimate particle sphericity and discriminate between liquid and ice particles. McFarquhar et al. (2013) proposed using area ratio, defined as the particle area divided by the area of the smallest circle bounding the particle, to classify the phase of particles with maximum dimensions between 35 and $60 \mu \mathrm{m}$. Using area ratio alone is the simplest technique for phase classification and has been implemented in the Earth Observing Laboratory's (EOL) OAP processing code. Throughout this study, we compare UWILD's performance with this technique, which we refer to simply as "area ratio". We include a schematic of area ratio in Fig. 1a.

A limitation of using area ratio alone is that quasispherical frozen particles, such as graupel, may be classified as liquid. Using particle surface roughness is an attractive alternative. Czys and Schoen Petersen (1992) fit a fourthdegree polynomial to quasi-spherical 2D particle images (with particle circumference represented in polar coordinates) to estimate surface roughness and distinguish between graupel particles and liquid drops. Other studies (Hunter et al., 1984; Moss and Johnson, 1994; Bower et al., 1996; Yang et al., 2016) have used Fourier analysis to quantify the sphericity and roughness of imaged particles and to classify them as liquid drops or frozen particles with particular habits. Holroyd (1987) also used a combination of particle shape and surface roughness to classify particle habit. They quantified surface roughness using the fine-detail ratio, defined as the perimeter multiplied by maximum dimension and divided by particle area, and used that and other particle properties to classify frozen hydrometeors into 10 habits, which we refer to as Holroyd habits. McFarquhar et al. (2018) modified this algorithm for use in mixed-phase clouds by classifying par- 


\section{Three Phase Discrimination Algorithms}

(a) Area Ratio
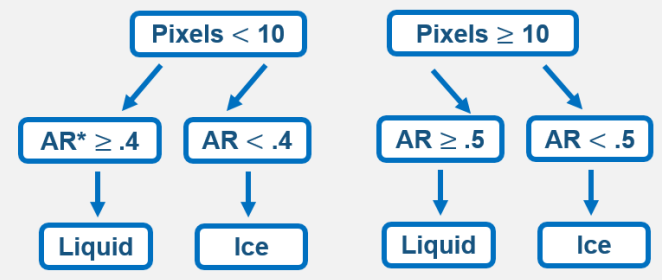

*AR = Area Ratio

(b)

Holroyd

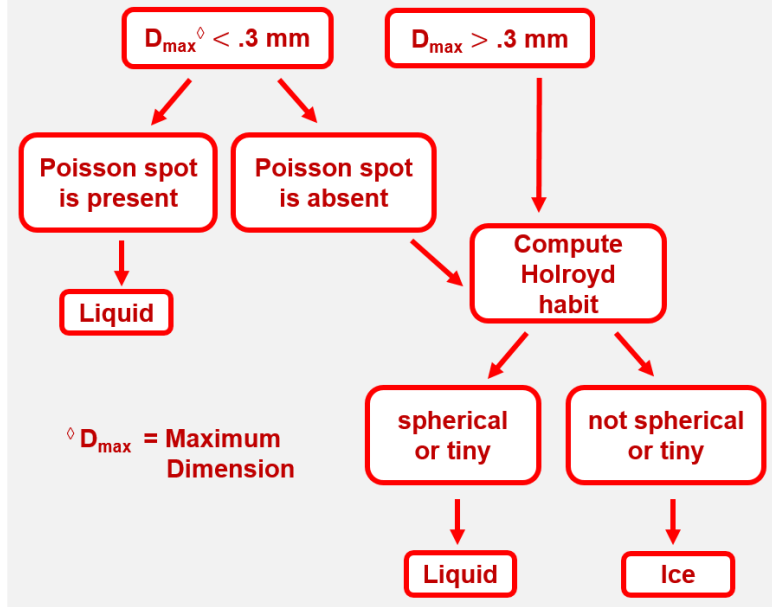

(c)

UWILD

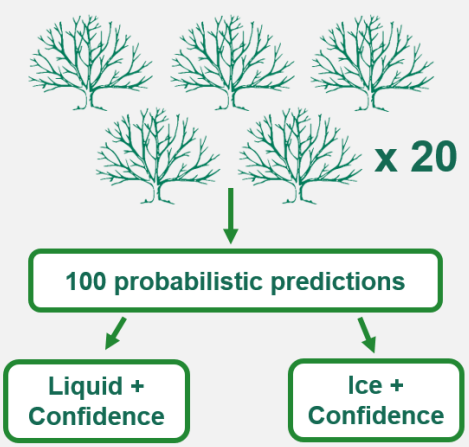

Figure 1. Schematic comparing the three-phase classification algorithms compared in this study. Using single-particle properties derived from the 2D-S probe as input, each panel describes the algorithm decision tree used to classify a particle as liquid or ice.

ticles labeled as tiny or spherical from the Holroyd (1987) scheme as liquid and all other particles as ice. Furthermore, for particles with maximum dimensions $<300 \mu \mathrm{m}$, the presence or absence of a light spot in the center of the particle, known as the Poisson spot (Arago and Gay-Lussac, 1819;
Heymsfield and Parrish, 1978), is recorded. If particles exhibit this diffraction pattern, they are classified as liquid and their Holroyd habit is not taken into account. This technique has been applied to SOCRATES data (Wu et al., 2019), and Wang et al. (2020) used the resulting phase classifications to compute number concentrations and water contents of droplets and frozen hydrometeors in SOCRATES-sampled clouds. Throughout this study, we compare UWILD's performance with this technique as well, which we refer to as "Holroyd" for simplicity, although it employs the Holroyd (1987) habit classification in conjunction with other techniques. We include a schematic of Holroyd in Fig. 1b.

Machine learning is attractive for single-particle phase classification because it allows for the use of multiple particle parameters without incurring the labor and time costs associated with handpicking thresholds for those parameters. Additionally, many machine learning algorithms produce both a classification and a model confidence for each particle, which can be regarded as the uncertainty in the phase classification for a well-calibrated model. Machine learning has been used to classify airborne particle probe images in many studies over the last decade (Lindqvist et al., 2012; Nurzynska et al., 2012, 2013; O'Shea et al., 2016; Praz et al., 2017, 2018; Xiao et al., 2019; Wu et al., 2020; Korolev et al., 2020; Touloupas et al., 2020), but to our knowledge, it has never been used to directly predict hydrometeor phase for binary OAP images.

The remainder of the article is arranged as follows. In Sect. 2, we describe the SOCRATES dataset and the development of our training, testing, and validation dataset. In Sect. 3, we introduce UWILD, and in Sect. 4, we evaluate UWILD and compare its performance with two preexisting phase classification algorithms. In Sect. 5, we show how concentrations of liquid and frozen hydrometeors vary with size and temperature, and we investigate sub- $1 \mathrm{~Hz}$ cloud phase heterogeneity in the SOCRATES dataset. A summary of UWILD and its implications for identifying particle phase in Southern Ocean clouds is discussed in Sect. 6.

\section{Processing SOCRATES data for single-particle phase classification}

\subsection{SOCRATES observations and description of the 2D-S}

Between 15 January and 25 February 2018, the US National Science Foundation (NSF) supported the SOCRATES campaign to sample diverse boundary layer clouds between Hobart, Tasmania, and the Antarctic continent (McFarquhar et al., 2020). SOCRATES employed the NSF-National Center for Atmospheric Research (NCAR) Gulfstream V (G-V) aircraft outfitted with three OAPs including the 2D-S, the Two-Dimensional Cloud (2DC) probe, and the Precipitation Imaging Probe (PIP). The 2DC and the PIP both suffered from quality control issues (Finlon et al., 2020), so we use 
the 2D-S particle images to develop single-particle phase classifications for the SOCRATES dataset. The SOCRATES campaign included $156-8 \mathrm{~h}$ flights. We use 14 out of the 15 SOCRATES flights, omitting RF15 due to an anomalously high occurrence of corrupted 2D-S particle images. Figure 2 shows the SOCRATES flight tracks and the distribution of $1 \mathrm{~Hz}$ in-cloud temperatures from the 14 SOCRATES flights used here. The majority $(85.5 \%)$ of the cloudy flight data occur within the temperature range that can support mixedphased clouds, -40 to $0^{\circ} \mathrm{C}$, while $15.1 \%$ of the cloudy flight data are warmer than $0{ }^{\circ} \mathrm{C}$, and the remaining $0.4 \%$ of the flight data are colder than $-40^{\circ} \mathrm{C}$.

The 2D-S uses a 128-element photodiode array in conjunction with high-speed electronics to generate shadowgraphs of particles with $10 \mu \mathrm{m}$ pixel resolution as they enter the instrument's sample volume (Lawson et al., 2006). Particle shadowgraphs, or images, are thus composed of $10 \mu \mathrm{m} \times 10 \mu \mathrm{m}$ square pixels. Particles with maximum dimensions smaller than $0.01 \mathrm{~mm}$ have areas smaller than that of a single pixel and cannot be detected by the 2D-S. Particles with maximum dimensions greater than $1.28 \mathrm{~mm}$ (the length of the photodiode array) have a higher likelihood of being partially cut off by the image buffer depending on their habit and orientation. The 2D-S has a sample volume between 10 and $16 \mathrm{~L} \mathrm{~s}^{-1}$ for typical SOCRATES aircraft speeds and can record compressed data at rates associated with particle concentrations up to about $100 \mathrm{~cm}^{-3}$ (Lawson et al., 2006), as they typically were during SOCRATES. We use the University of Illinois/Oklahoma OAP Processing Software (UIOOPS; McFarquhar et al., 2018) to compute particle properties of individual particles from the horizontal channel of the 2D-S. Due to optical limitations of the 2D-S, quasi-spherical outof-focus particles exhibiting Poisson spots are size-corrected following Korolev (2007). We apply several criteria to filter particles for use in this study. First, we only use particles whose center is within the 2D-S field of view to minimize uncertainties in determining the reconstructed particle size (Heymsfield and Baumgardner, 1985; Field, 1999). Due to uncertainties in defining the probe's depth of field and sample area (Heymsfield and Parrish, 1978; Baumgardner and Korolev, 1997; Jackson et al., 2012) and limited shape information to support robust classification for smaller particles (Korolev et al., 1991; Baumgardner et al., 2017), particles with fewer than 25 pixels are excluded from this study. Because of this, there are no particles classified as tiny (defined as $<25$ pixels) using the Holroyd scheme (Fig. 1a), and only the right subtree of area ratio (Fig. 1b) is relevant. Throughout this study, we will use area-equivalent diameter $\left(D_{\text {eq }}\right)$ to represent particle size. For the $2 \mathrm{D}-\mathrm{S}, D_{\text {eq }}$ in millimeters is computed from the number of pixels as follows:

$D_{\text {eq }}=2 \times 10^{-2} \times \sqrt{\text { number of pixels } \times \pi^{-1}}$.

We use 15 particle features, listed in Table 1, to train our machine learning model. These 15 features represent a sub- set of all of the particle properties that UIOOPS computes. We visually define the particle features using example particles in Fig. A1, and we show histograms of the particle features for the SOCRATES dataset in Fig. A2. We use the common logarithm of inter-arrival time $\left(\log _{10}\right.$ (iat)), which is the time elapsed since the previous particle was imaged within the 2D-S sample volume, instead of the absolute interarrival time because machine learning models are optimized to train on normally distributed variables. Inter-arrival time is the only feature that is substantially non-normally distributed and requires normalization. We show distributions of $\log _{10}$ (iat) for liquid and ice-dominated particles from our training dataset in Fig. 3a. We discuss how we prepare our training dataset in Sect. 2.2. The clear separation in the peaks of the two distributions, with liquid particles associated with smaller inter-arrival times, indicates that inter-arrival time is useful for phase classification. The distribution for the icedominated particles is bimodal, and the smaller mode in the smaller inter-arrival times may be a signature of shattered artifacts (Korolev et al., 2013). These suspected shattered artifacts with inter-arrival times $<10^{-5} \mathrm{~s}$ represent $7 \%$ of the ice-dominated particles and are thus expected to minimally affect how UWILD uses inter-arrival time for phase discrimination. We retrained UWILD with $\log _{10}$ (iat) excluded as a sensitivity test and found that UWILD's skill in identifying small ice particles sharply decreased, while its skill at identifying other particles decreased only slightly. This result is described in greater detail in Sect. 4.

\subsection{Preparation of training, validation, and test data}

Classification problems are a type of supervised machine learning that require a dataset with known classifications to use for model training, cross-validation during hyperparameter (i.e., model configuration parameter) tuning, and model testing. Creating this dataset, hereafter referred to as the TTV (for train-test-validate) set, is the biggest challenge associated with this particular machine learning problem. Using manual inspection to build a TTV set, as most studies using machine learning for particle image classification have done, would limit the scope of our TTV set to particles large enough to identify by eye that have an unambiguous phase. This would result in our TTV set being substantially different from a set of randomly sampled particles from SOCRATES and would thus reduce the generalizability of our machine learning model for the whole SOCRATES dataset. Instead, we use in situ flight data, including temperature from the Harco heated total air temperature sensor (EOL, 2019), water vapor mixing ratio from the Vertical-Cavity Surface-Emitting Laser (VCSEL) hygrometer (Zondlo et al., 2010; Diao, 2021), and voltage from the Rosemount Icing Detector (RICE; EOL, 2019), to identify flight periods where the hydrometeors are most likely to be all or mostly the same phase. 
(a) SOCRATES Flight Tracks
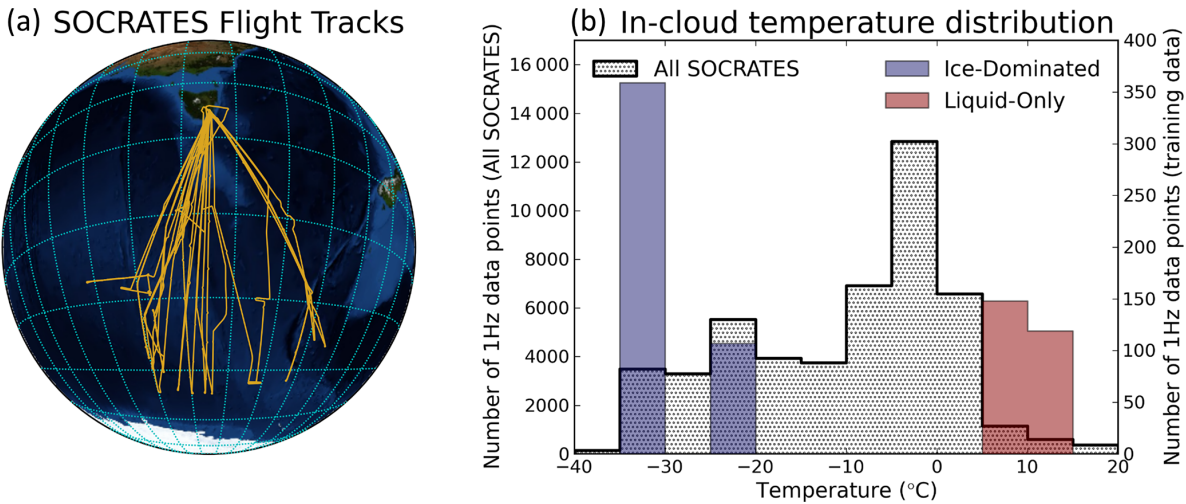

Figure 2. (a) SOCRATES flight tracks from RF01-RF14 (the flights analyzed in this study); (b) $1 \mathrm{~Hz}$ in-cloud temperature histograms for RF01-RF14 (hatched, left $y$ axis), with ice-dominated data from the TTV set (blue, right $y$ axis) and liquid-only data from the TTV set (red, right $y$ axis). In-cloud data include all samples with at least one particle that satisfy our selection criteria (described in Sect. 2.1).
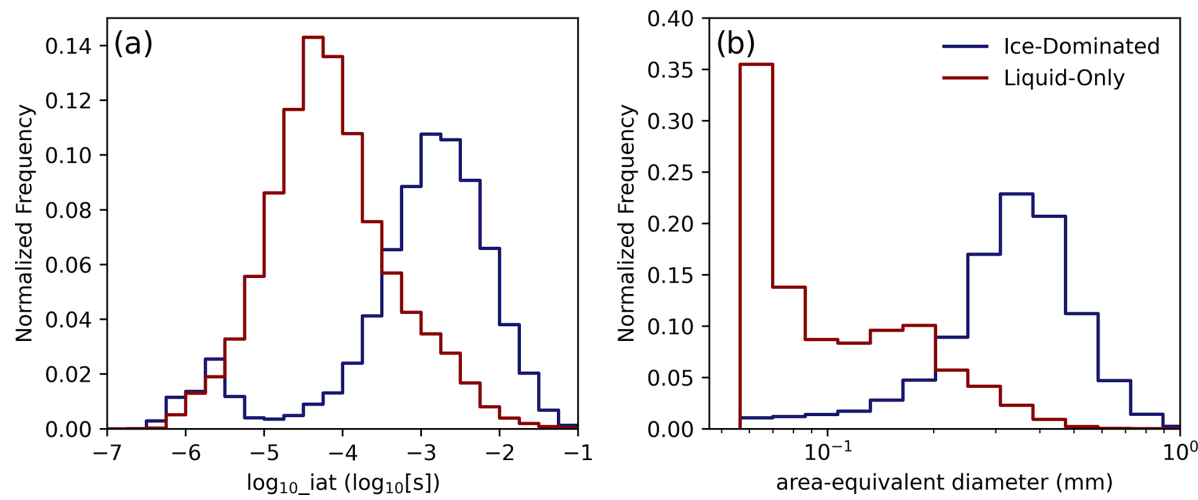

Figure 3. Distributions of the common logarithm of inter-arrival time (a) and particle size (b) for the liquid-only (red) and ice-dominated (blue) data from the TTV set.

The RICE is an oscillating probe that is sensitive to mass buildup from supercooled drops that have frozen onto its sensing cylinder. When supercooled water is present at temperatures lower than $-5^{\circ} \mathrm{C}$ with a mass concentration above the theoretical detection limit of $0.025 \mathrm{~g} \mathrm{~m}^{-3}$ (D'Alessandro et al., 2021), sufficient ice builds up on the sensing cylinder to decrease its oscillating frequency, which is converted to a positive voltage. Thus, a changing RICE voltage indicates the presence of supercooled water. A near-constant RICE voltage indicates that supercooled water is not present in a sufficient quantity to trigger an instrument response.

Equations of saturation pressure with respect to liquid and ice (Murphy and Koop, 2005) were used to calculate relative humidity with respect to liquid $(\mathrm{RH})$ and ice $\left(\mathrm{RH}_{\mathrm{i}}\right)$. Uncertainties in $\mathrm{RH}$ and $\mathrm{RH}_{\mathrm{i}}$ can be derived based on the uncertainties associated with temperature and water vapor. Uncertainties range from $6.4 \%$ to $6.8 \%$ for $\mathrm{RH}$ and from $6.5 \%$ to $6.9 \%$ for $\mathrm{RH}_{\mathrm{i}}$, from 0 to $-40{ }^{\circ} \mathrm{C}$, respectively. Identifying liquid phase regions of clouds is simple because frozen hydrometeors rarely persist at temperatures above $5{ }^{\circ} \mathrm{C}$ (Yuter et al., 2006; Oraltay and Hallett, 2005). Thus, we select a
5 min flight period where the temperature varies between 6 and $12{ }^{\circ} \mathrm{C}$ as a liquid-only period. We show time series of $1 \mathrm{~Hz}$ temperature, $\mathrm{RH}$, particle count, and liquid fraction from UWILD for this region in Fig. 4a. There is only RH data available towards the end of the period, and the $\mathrm{RH}$ is close to $100 \%$ there. UWILD classifies most particles as liquid throughout the flight period, but its accuracy decreases towards the end of the period. We explain how UWILD classifies particles in Sect. 3, and we quantify UWILD's performance and identify biases in its classifications in Sect. 4. A histogram of temperature for the liquid-only period is shown in Fig. 2b, and normalized histograms of $D_{\text {eq }}$ and $\log _{10}$ (iat) are shown in Fig. 3. Small particles $(<100$ pixels or $D_{\text {eq }}<0.1 \mathrm{~mm}$ ) dominate the liquid-only dataset.

Supercooled water can persist at all temperatures above the homogeneous nucleation threshold of $-40{ }^{\circ} \mathrm{C}$ (Korolev et al., 2017) and below $0^{\circ} \mathrm{C}$. Since there are no multi-second periods of in-cloud data from SOCRATES with temperatures below $-40^{\circ} \mathrm{C}$ (Fig. $2 \mathrm{~b}$ ), we cannot be certain that all particles within any given SOCRATES flight period are frozen. However, we can use the aforementioned atmospheric pa- 
Table 1. Description of particle features used in UWILD. Features have units of numbers of pixels except where different units are specified.

\begin{tabular}{ll}
\hline Particle feature & Description [units] \\
\hline $\begin{array}{l}\text { length } \\
\text { width }\end{array}$ & Pixels in time dimension \\
area & Pixels in photodiode dimension \\
perimeter & Projected area from the number of shadowed pixels (1 pixel $\left.=0.0001 \mathrm{~mm}^{2}\right)\left[\mathrm{mm}^{2}\right]$ \\
max_dimension & Perimeter following the particle boundary [mm] \\
area_equivalent_diameter & Maximum dimension of the smallest circle bounding the particle [mm] \\
area_ratio & Diameter of a circle with an area equal to the particle area [mm] \\
percent_shadow_area & Ratio between particle area and the area of the smallest circle bounding the particle \\
touching_edge & Ratio between particle area and the length $\times$ width \\
& Binary depicting whether the particle is entirely within the photodiode array [0] or touches an edge \\
max_top_edge_touching & of the array [1] \\
max_bottom_edge_touching & Maximum number of times the top diode is shadowed in succession \\
edge_at_max_hole & Maximum number of times the bottom diode is shadowed in succession \\
& Number of pixels between the top and bottom edges of the particle for the slice containing the \\
max_hole_diameter & largest gap inside the particle \\
fine_detail_ratio & Diameter of the largest hole inside the particle \\
logidiat) & Ratio between perimeter $\times$ maximum dimension and particle area following Holroyd (1987) \\
& Log of the inter-arrival time between particles [log 10 (s)]
\end{tabular}
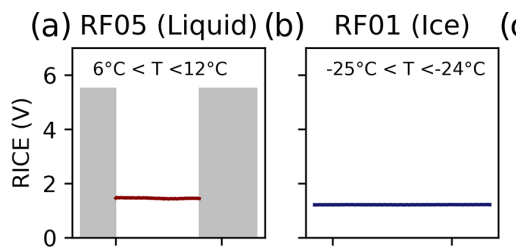

(c) RF04 (Ice)

(d) RF12 (Mixed) (e) RF03 (Mixed)
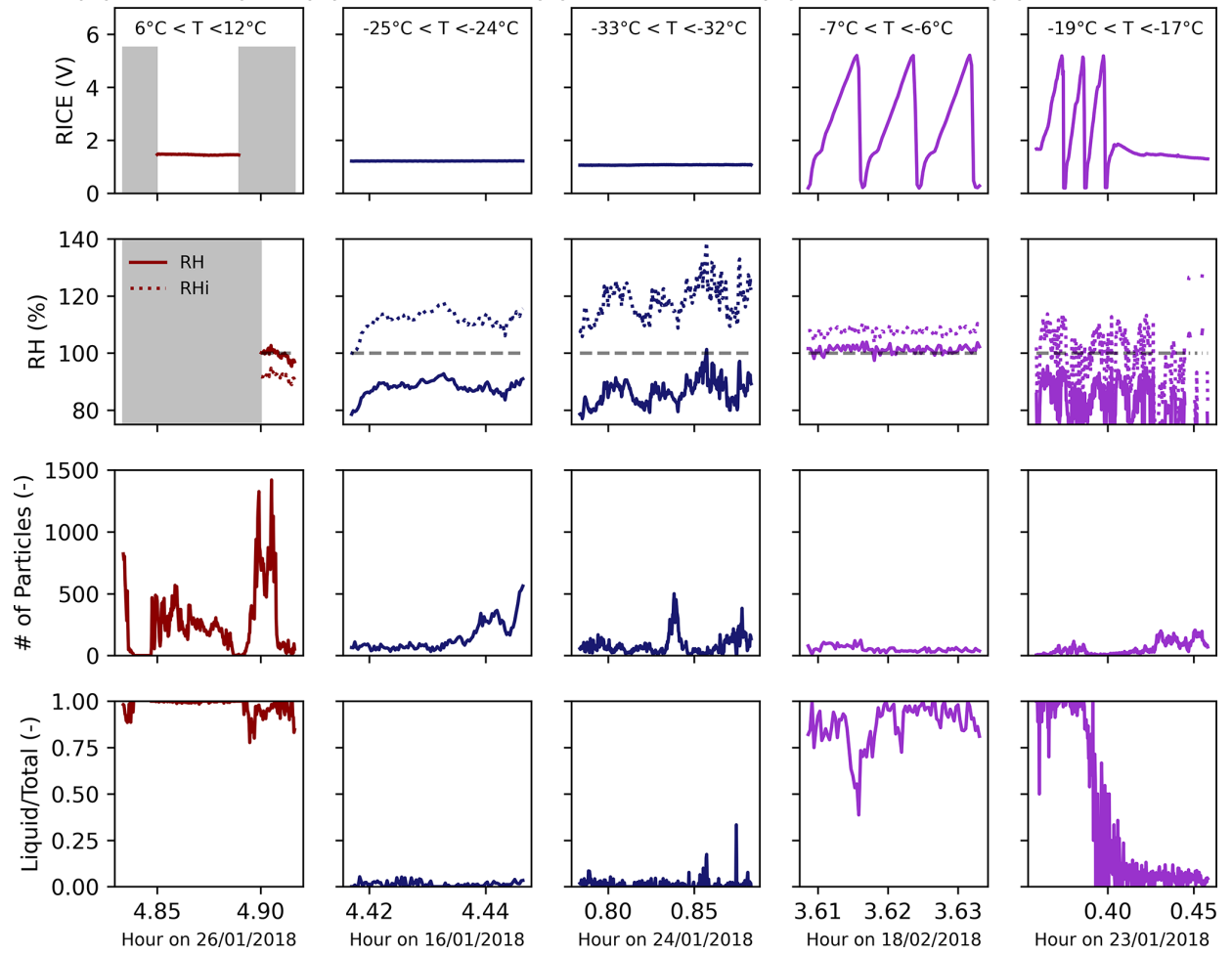

Figure 4. Time series of atmospheric parameters and particle properties for the liquid-only period (a), the two ice-dominated periods (b, c), and the two example mixed-phase periods (d, e). Voltage from the RICE (row 1), relative humidity with respect to liquid (solid line) and ice (dashed line) (row 2), the number of particles that satisfy our selection criteria (row 3), and liquid fraction as determined by the UWILD algorithm (row 4) are shown for each flight period. A RICE response is expected when the supercooled water content exceeds $0.025 \mathrm{~g} \mathrm{~m}{ }^{-3}$. Missing data are indicated with grey. The temperature range sampled in each period is shown on the plot of RICE voltage in the top row. 
rameters and particle probe images to identify periods where we have very high confidence that over $99 \%$ of the particles are frozen. We refer to these as ice-dominated periods. We use temperature, RH with respect to ice and liquid water, voltage from the RICE, and particle images from the 2D-S to identify two ice-dominated periods, from flights RF01 and RF04, which we show in Fig. $4 \mathrm{~b}$ and c, respectively. The icedominated periods are defined as having no RICE response, are subsaturated with respect to liquid, and are supersaturated with respect to ice. A RICE response, which consists of the $1 \mathrm{~Hz}$ voltage oscillating over a $20 \mathrm{~s}$ period, is expected when the supercooled water content exceeds $0.025 \mathrm{~g} \mathrm{~m}^{-3}$. RF04 is the source of $70 \%$ of particles in the combined icedominated dataset, and RF01 is the source of the remaining particles. A histogram of temperature for the combined ice-dominated dataset is shown in Fig. 2b, and normalized histograms of $D_{\text {eq }}$ and $\log _{10}$ (iat) for the same dataset are shown in Fig. 3. The ice-dominated dataset is composed primarily of medium-sized and large particles $(\geq 100$ pixels or $D_{\text {eq }} \geq 0.1 \mathrm{~mm}$ ).

We manually inspected 1000 2D-S particle images from the combined ice-dominated dataset with $0.2 \mathrm{~mm}<D_{\text {eq }}<$ $0.8 \mathrm{~mm}$, which account for $87 \%$ of the particles in the icedominated dataset, and found that $0.6 \%$ of the particles are likely liquid. Thus, if UWILD classified all particles in the ice-dominated region correctly, it would have a slightly higher performance than what is reported here (Sect. 4) because we compute performance metrics assuming that all particles in the ice-dominated region are frozen.

The SOCRATES payload also included the Particle Habit Imaging and Polar Scattering (PHIPS) probe, which records high-quality images of particles with a maximum imaging rate set at $3 \mathrm{~Hz}$ for SOCRATES and measures particle scattering phase functions at a maximum rate of $3.5 \mathrm{kHz}(\mathrm{Ab}-$ delmonem et al., 2016; Schnaiter et al., 2018). The PHIPS dataset (Schnaiter, 2018b) includes manual classifications of particle phase based on particle images and automated classifications of particle sphericity based on particle scattering phase functions (Waitz et al., 2021). Because the maximum scattering phase function data acquisition rate is greater than the maximum imaging rate, there are more automated classifications than manual classifications. Particle sphericity is a good indicator of particle phase for small and medium-sized particles. However, large liquid drops are typically aspherical or elongated because they are distorted due to pressure differences in the instrument's inlet, as discussed in Supplement 4 of Waitz et al. (2021).

We examine manual and automated classifications for the PHIPS to further evaluate our liquid-only and ice-dominated periods. The PHIPS automated classification algorithm identified 132 particles as spherical and 45 particles as aspherical during our liquid-only period, while manual classifications of 106 images are universally liquid. While the PHIPS was not available for RF01, the PHIPS algorithm automatically classified 3905 particles as aspherical and only 4 particles as spherical during our ice-dominated period from RF04. Manual classifications of 320 images are universally frozen.

We compare the liquid-only period and two ice-dominated periods with two examples of mixed-phase periods in Fig. 4. The first mixed-phase period (Fig. 4d) samples a stratocumulus cloud within the boundary layer. The RICE voltage oscillates throughout the period, and the liquid fraction is greater than $75 \%$ most of the time. The cloud is saturated with respect to liquid. The second mixed-phase period (Fig. 4e) sampled near the top of altostratus cloud. This period is colder and, on average, subsaturated with respect to liquid water and saturated with respect to ice. The RH is subsaturated even though the cloud is liquid-dominated in the sampled region because the aircraft is skirting a horizontally variable cloud top. The liquid fraction is close to 1.0, and the RICE voltage oscillates until 00:23:00 UTC ( $\sim 0.4$ UTC), after which the liquid fraction decreases abruptly, and the RICE voltage stabilizes. The change in phase occurs because the aircraft is initially sampling the cloud top and transitions to sampling below the cloud top.

The liquid-only period includes 90000 particles that pass our size threshold, while the ice-dominated periods include 55000 particles in total. All particles drawn from the liquidonly period are labeled liquid, and all particles drawn from the ice-dominated periods are labeled ice. These labels are taken as truth for the purposes of model training and evaluation (Sect. 4). We partition the particles into three size categories: small (corresponding to 25-99 pixels or $D_{\text {eq }}$ of approximately $0.056-0.1 \mathrm{~mm})$, medium (100-699 pixels or $0.1-0.3 \mathrm{~mm}$ ), and large (> 700 pixels or $>0.3 \mathrm{~mm}$ ). In the remainder of this study, all references to particle size will be in terms of $D_{\text {eq }}$. We then randomly subsample the liquid particles down to have an equal total number of ice and liquid particles, preserving the ratio between the three size categories for each phase separately. These particles are then partitioned into training $(60 \%)$, test $(20 \%)$, and validation (20\%) sets, again preserving the original ratios between the three size categories for each phase separately and including an equal number of particles from each phase, in each set. We refer to the combined training, test, and validation sets as the TTV set. We explicitly preserve these rough size distributions to ensure that the test set has a reasonable number of small ice crystals and large liquid drops for evaluation, as these particles are rare enough in the full TTV dataset that a completely random partition risks having them undersampled in the test set. Having the same number of liquid and ice particles in the test set simplifies interpretation of model performance summary statistics, as discussed at the beginning of Sect. 4. The composition of the TTV set is broken down in Table 2. We show histograms of particle features from the TTV set and from the whole SOCRATES dataset (14 flights) in Fig. A2, and we discuss out of sample particles in Appendix A. 
Table 2. Number of particles by size class in the training, validation, and test sets.

\begin{tabular}{llrrc}
\hline Phase & Particle size class & Training & Validation & Test \\
\hline \multirow{2}{*}{ Ice } & Small $\left(0.056 \mathrm{~mm} \leq D_{\text {eq }}<0.1 \mathrm{~mm}\right)$ & $1.7 \mathrm{k}$ & $0.59 \mathrm{k}$ & $0.6 \mathrm{k}$ \\
& Medium $\left(0.1 \mathrm{~mm} \leq D_{\text {eq }}<0.3 \mathrm{~mm}\right)$ & $12.6 \mathrm{k}$ & $4.2 \mathrm{k}$ & $4.2 \mathrm{k}$ \\
& Large $\left(D_{\text {eq }} \geq 0.3 \mathrm{~mm}\right)$ & $18.6 \mathrm{k}$ & $6.2 \mathrm{k}$ & $6.2 \mathrm{k}$ \\
\hline \multirow{2}{*}{ Liquid } & Small $\left(0.056 \mathrm{~mm} \leq D_{\text {eq }}<0.1 \mathrm{~mm}\right)$ & $20.1 \mathrm{k}$ & $6.7 \mathrm{k}$ & $6.7 \mathrm{k}$ \\
& Medium $\left(0.1 \mathrm{~mm} \leq D_{\text {eq }}<0.3 \mathrm{~mm}\right)$ & $11.4 \mathrm{k}$ & $3.8 \mathrm{k}$ & $3.8 \mathrm{k}$ \\
& Large $\left(D_{\text {eq }} \geq 0.3 \mathrm{~mm}\right)$ & $1.5 \mathrm{k}$ & $0.5 \mathrm{k}$ & $0.5 \mathrm{k}$ \\
\hline
\end{tabular}

\section{UWILD: description and interpretation}

A key consideration for all machine learning applications is the choice of machine learning model. One approach to analyzing particle probe images is to apply deep learning directly to the captured image (e.g., Xiao et al., 2019; Touloupas et al., 2020; Wu et al., 2020; Korolev et al., 2020). Here, we take a simpler approach and employ a random forest model (Breiman, 2001; Pedregosa et al., 2011), which requires a preprocessing step to extract relevant image features (e.g., particle area or perimeter; full list in Table 1). Classification is then carried out using these features as inputs (Lindqvist et al., 2012; Nurzynska et al., 2012, 2013; O'Shea et al., 2016; Praz et al., 2018, 2017). An advantage of this approach is that it simplifies the inclusion of features not directly related to particle appearance; in particular, we show that inter-arrival time is a valuable discriminator of liquid and ice particles. Random forests can also provide more interpretable results, as the trained model can be analyzed to investigate relative feature importance. Another advantage (shared by many machine learning approaches) is the determination of classification confidence, which can be useful in filtering out more uncertain classifications, or estimating uncertainties in calculated bulk properties such as liquid water content.

For a decision tree trained using a supervised learning approach, the training set is split by thresholding features (e.g., whether area ratio is more or less than 0.8 ); precisely which feature and which value is determined by whatever "best" splits the dataset into distinct categories (for UWILD the max Gini impurity reduction criterion is used). This process is repeated on each data subset until the data are entirely partitioned into distinct categories. In a random forest, multiple such trees (100 for UWILD) are trained using random subsets of data features. Randomness is introduced here to reduce overfitting to the training set and improve model generalizability. For a given test data point, each tree provides a classification, and the plurality vote of all trees is the overall category assigned to the data point, with the proportion of trees voting for that category as the model confidence. We include a simple schematic of UWILD in Fig. 1c.

A model is well-calibrated if its model confidence (internal prediction probability) accurately reflects its performance.

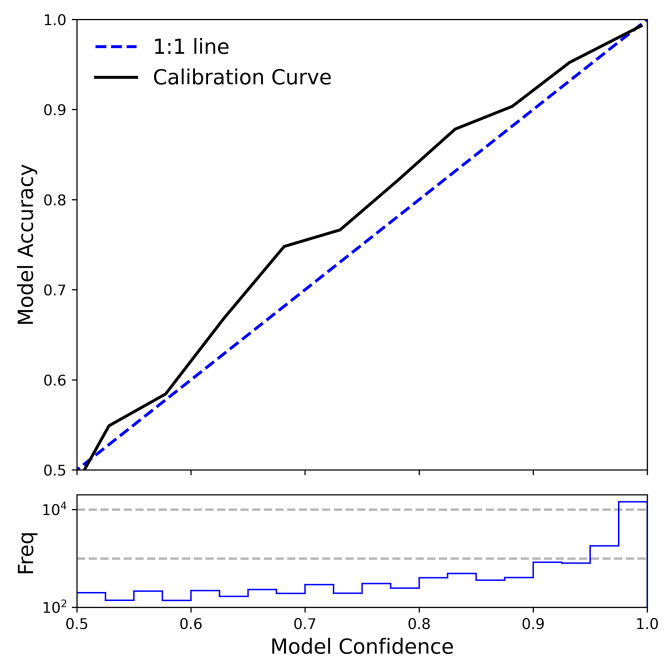

Figure 5. UWILD confidence is plotted against UWILD accuracy with a black solid line (this is referred to as the calibration curve) in the top row, and a histogram of model confidence is shown in the bottom row on a shared $x$ axis. This analysis is done on the test set. The proximity between the calibration curve and the one-to-one blue dashed line implies that UWILD confidence can be used as a proxy for the uncertainty in UWILD's classifications.

Figure 5 shows this relationship between model confidence (from the random forest votes) and model accuracy (how likely the model was to correctly classify particles), evaluated on the test set. A one-to-one relationship is ideal because it indicates that we can directly use model confidence as an estimate of prediction uncertainty. For example, a particle classified as ice with a model confidence of $75 \%$ should be seen as $75 \%$ likely to be ice, and $25 \%$ likely to be liquid. Figure 5 also shows that UWILD has a confidence of $95 \%$ or higher for $73 \%$ of the particles in the TTV set.

To better understand how the UWILD classifier determines particle phase, we quantify how much it relies on each of the 15 different features (listed in Table 1) using permutation feature importance analysis. This technique measures how much a model relies on the information encoded in a particular feature by calculating model accuracy on a test set, randomly shuffling a given feature, and measuring how much the accuracy decreases. The random shuffling of a fea- 


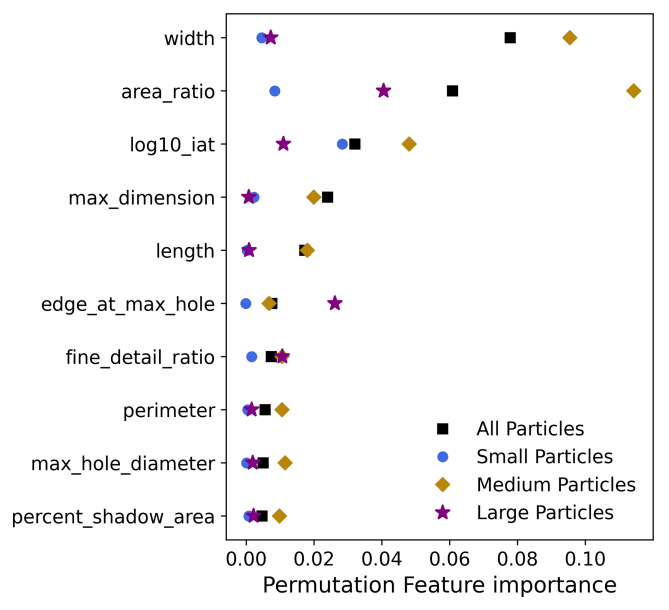

Figure 6. Permutation feature importance for the 10 most important features is shown for all particles (black squares) and for the 3 size classes separately. Features are shuffled 10 times, and the mean feature importance from the 10 trials is shown here.

ture renders that feature useless to the model classification, and the model accuracy will decrease substantially for a very significant feature. This analysis can be rapidly performed multiple times for each feature. Another advantage of permutation feature importance is that it is a function of the dataset being used for evaluation as well as the model, so it can be calculated separately for different subsets of the data or for entirely new test datasets. Other measures of feature importance (such as impurity-based feature importance) are functions only of the model and do not share this advantage. A relevant drawback to all measures of feature importance is that they are affected by correlations between features. As correlated features share information, model performance may not decrease as much when a particular feature is shuffled, as a (previously) correlated different feature may still encode the relevant information. However, decorrelating variables prior to use, which would address this issue, complicates model interpretation (while not significantly affecting model performance), and so we chose to preserve original features and caution against too minute a dissection of the permutation feature importance.

Figure 6 shows the permutation feature importance of the top 10 features, split by particle size and evaluated on the model test set. Overall, we see that width, area ratio, and $\log _{10}$ (iat) are the most important. The next two features (max dimension and length) both encode size and correlate well with width, while the remaining features have low impact on model accuracy. Put another way, the model primarily relies on these first three features for classification. Considering differences between size classes, we note that width and all other size-related features are most important for medium particles, which is to be expected, as larger particles are predominantly ice, and smaller particles are predominantly liquid, with medium particles varying the most. For small parti- cles, $\log _{10}$ (iat) is most important, and for large particles, area ratio is most important (likely because small and medium particles are mostly quasi-spherical irrespective of phase). Regarding correlated features, the results in Fig. 6 should not be taken to mean that width in particular is a key discriminator as opposed to length or max dimension but rather that width is a good estimator of particle size, which is the particle characteristic that matters in determining its phase. If particle width were removed from the feature set, then another size-encoding feature would appear more important.

\section{Comparison between phase classification schemes}

For quantitative evaluation of a classification model, an intuitive summary metric is model accuracy (the ratio of correct classifications to total classifications). The overall accuracy of UWILD, Holroyd, and area ratio on our test set is $94.9 \%, 78.5 \%$, and $71.8 \%$, respectively, indicating that UWILD is performing quite well. However, accuracy is most suitable for balanced classification problems (i.e., when data are spread evenly across categories). In the case of highly unbalanced problems, high accuracy can be achieved by systematically erring in favor of the dominant category. For example, small particles in the test set are overwhelmingly liquid (Fig. 3b), so high accuracy can be achieved by predicting that all small particles are liquid at the expense of correctly classifying small ice particles.

Model performance, especially for unbalanced classification problems, can be better measured by calculating precision (the ratio of all particles correctly classified as liquid to all particles classified as liquid) and recall (the ratio of all particles classified as liquid to all true liquid particles). Both scores range from $0-1$, and they penalize false positives and false negatives, respectively, for a particular category. These scores are unified in the $F_{1}$ score, which is their harmonic mean:

$F_{1}=\frac{2 \times \text { precision } \times \text { recall }}{\text { precision }+ \text { recall }}$.

The $F_{1}$ score is a conservative measure of model performance because the lesser of recall and precision will dominate the harmonic mean, and it can be calculated for various data subsets. We show the $F_{1}$ scores for Holroyd, area ratio, and UWILD in Fig. 7 as a function of phase and size class. This analysis is performed on our test set. UWILD outperforms Holroyd and area ratio for all phases and size classes. It has the best performance for small liquid $\left(F_{1}=0.982\right)$ and large ice $\left(F_{1}=0.992\right)$ particles and performs less well with small ice $\left(F_{1}=0.765\right)$ and large liquid $\left(F_{1}=0.893\right)$ particles. While UWILD performs least well when classifying small ice and large liquid, it nevertheless has a particularly large performance advantage over Holroyd and area ratio for those categories. Holroyd outperforms area ratio for medium and large ice particles, whereas area ratio outperforms Holroyd for small ice particles and liquid particles of all sizes. 
We tested the sensitivity of UWILD's performance to the inclusion of $\log _{10}$ (iat) by retraining UWILD without it. We found that the $F_{1}$ score for small ice particles dropped from 0.765 to 0.475 , whereas the $F_{1}$ scores for the other phase and size classes dropped only slightly.

The TTV set only includes ice particles occurring at the lowest temperatures $\left(<-23^{\circ} \mathrm{C}\right)$ sampled during SOCRATES, which may have different average ice properties than ice crystals occurring at higher temperatures. To account for this, we generated a hand-labeled dataset to evaluate UWILD's ability to detect ice crystals at higher temperatures. We manually classified 1000 randomly sampled images with $0.2<D_{\text {eq }}<0.8 \mathrm{~mm}$, occurring at temperatures higher than $-23^{\circ} \mathrm{C}$. The resulting dataset contained 861 ice particles, 78 liquid particles, and 61 ambiguous particles (which were discarded). We found that UWILD, Holroyd, and area ratio had $F_{1}$ scores of $0.63,0.44$, and 0.26 , respectively. For UWILD, broken down by phase, the $F_{1}$ scores were 0.97 (ice) and 0.63 (liquid). Thus, UWILD still outperforms the other two algorithms despite all three exhibiting lower $F_{1}$ scores for the hand-labeled set. This lower performance is consistent with lower model confidences for this size and temperature range, as discussed below. In the rest of this section, we identify differences between the algorithms and biases within each algorithm to explain the discrepancies in their performances. We use the whole SOCRATES dataset (14 flights), which includes 5.76 million classified 2D-S images, for our analysis from hereon.

Table 3 shows how many particles each algorithm classified as liquid and ice for each size class and in total. In general, Holroyd classifies the most particles as ice, and area ratio classifies the most particles as liquid. UWILD and area ratio both classify over $90 \%$ of the small particles as liquid, whereas Holroyd classifies only $70 \%$ of them as liquid. Area ratio classifies three-quarters of the medium particles and half of the large particles as liquid. In contrast, the other two algorithms classify about $40 \%$ of the medium particles and $0 \%$ (Holroyd) to $2.7 \%$ (UWILD) of the large particles as liquid.

In Fig. 8, we show the fraction of particles classified as liquid, from the three-phase discrimination algorithms, in the phase spaces of temperature vs. particle size (left column) and RH vs. particle size (right column). In the first row, we show a 2D histogram of the number of classified particles; in the second row, we show a 2D histogram of the confidence from UWILD; in the third, fourth, and fifth rows, we show 2D histograms of the fraction of particles classified as liquid by the three-phase discrimination algorithms. At temperatures greater than $-20^{\circ} \mathrm{C}$, UWILD confidence is lowest in areas where UWILD transitions between having a high liquid fraction and a low liquid fraction (Fig. 8b). UWILD confidence is also low for small particles at temperatures below $-20^{\circ} \mathrm{C}$, which can have high or low liquid fractions. All three algorithms show a decrease in liquid fraction for small particles at temperatures between -20 and $-30{ }^{\circ} \mathrm{C}$ and an increase in the liquid fraction at temperatures below $-30^{\circ} \mathrm{C}$ (Fig. 8c-e). This behavior is a consequence of small sample size, as the liquid-dominated data below $-30^{\circ} \mathrm{C}$ come from just one flight that sampled the top of an altostratus cloud, whereas the ice-dominated data at higher temperatures come from several flights that sampled the middle of altostratus clouds.

Since temperature and RH are not inputs to any of the algorithms, we can use them to gauge whether the particle classifications make physical sense. In other words, we can use these atmospheric parameters to make broad predictions of hydrometeor phase and determine which algorithm is most consistent with these predictions. We expect that small particles will be entirely liquid above $0{ }^{\circ} \mathrm{C}$ and that large particles will be primarily liquid above $0{ }^{\circ} \mathrm{C}$ and entirely liquid above $5^{\circ} \mathrm{C}$ due to having longer melting timescales (Oraltay and Hallett, 2005). Furthermore, we expect that if the liquid fraction is not already 1.0 at $0{ }^{\circ} \mathrm{C}$, then it will increase as temperature increases above $0^{\circ} \mathrm{C}$, for any particle size. We note that the abrupt disappearance of particles with $D_{\text {eq }}>0.5 \mathrm{~mm}$ at $0^{\circ} \mathrm{C}$ is a signature of ice particles melting.

Ice and liquid precipitation formation mechanisms have been observed to operate simultaneously at temperatures as low as $-28^{\circ} \mathrm{C}$ (Huffman and Norman, 1988; Cober et al., 2001b; Kajikawa et al., 2000; Korolev et al., 2002; Silber et al., 2019), so we cannot use temperature alone to make a prediction for the liquid fraction of particles at temperatures below $0{ }^{\circ} \mathrm{C}$. However, we do expect to see a size dependence in the liquid fraction. To our knowledge, the largest liquid particle associated with supercooled drizzle formation (as opposed to melting frozen hydrometeors) that has been noted in the literature has a maximum dimension of $0.625 \mathrm{~mm}$ (Cober et al., 2001b). Most SOCRATES data were collected in conditions that could not support the relofting of melted frozen hydrometeors. Furthermore, melted frozen hydrometeors are rarely lofted to temperatures below $-5^{\circ} \mathrm{C}$ in environments that do support relofting (Oraltay and Hallett, 2005). Thus, we expect that medium-sized and large droplets at temperatures below $-5^{\circ} \mathrm{C}$ are primarily formed via supercooled drizzle formation and will not be present at the largest sizes $(0.625-1 \mathrm{~mm})$.

Holroyd and UWILD classify many medium-sized $\left(0.1 \mathrm{~mm}<D_{\text {eq }}<0.3 \mathrm{~mm}\right)$ and large $\left(D_{\text {eq }}>0.3 \mathrm{~mm}\right)$ particles as ice at high temperatures $\left(>0^{\circ} \mathrm{C}\right)$. Liquid fractions for Holroyd sharply decrease to near 0.0 for particles with $D_{\text {eq }}$ between 0.2 and $0.3 \mathrm{~mm}$ at all temperatures (Fig. 8e). This strong size dependence arises from the fact that Holroyd only considers the presence or absence of a Poisson spot for particles with maximum dimensions less than $0.3 \mathrm{~mm}$ (Fig. 1b). Particles exceeding that maximum dimension threshold must be nearly spherical in shape to be classified as liquid because the presence of a Poisson spot is not factored into the phase classification. A near- 0.0 liquid fraction for Holroyd for $D_{\text {eq }}>0.3 \mathrm{~mm}$ is unrealistic for temperatures above $0{ }^{\circ} \mathrm{C}$, where the liquid fraction should increase with temperature. 

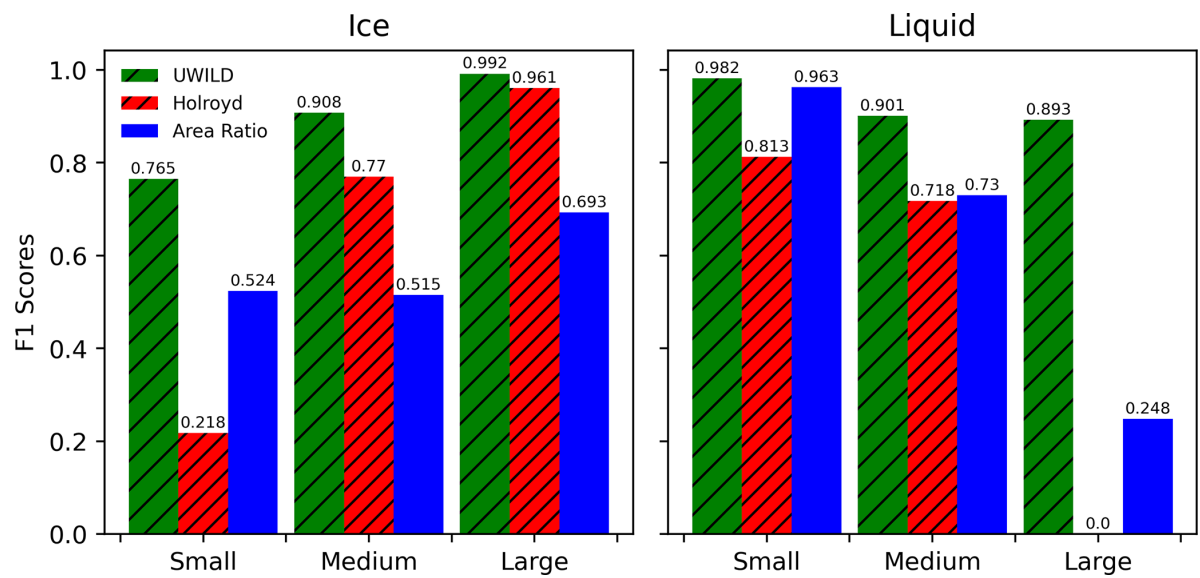

Figure 7. $F_{1}$ scores are shown for UWILD (green), Holroyd (red), and area ratio (blue) for different size classes. $F_{1}$ scores for small and medium particles may be slightly underestimated due to the presence of liquid drops in the ice-dominated TTV set.

Table 3. Total particles and number of particles classified as liquid from the three-phase discrimination algorithms for each size class. Numbers in parentheses are the percentage of total particles classified as liquid. SOCRATES flights RF01-RF14 are used.

\begin{tabular}{lrrrr}
\hline Particle size class & Total & UWILD & Holroyd & Area ratio \\
\hline Small $\left(0.056 \mathrm{~mm} \leq D_{\text {eq }}<0.1 \mathrm{~mm}\right)$ & 2788219 & $2641062(94.7)$ & $1867682(70.0)$ & $2563437(91.9)$ \\
Medium $\left(0.1 \mathrm{~mm} \leq D_{\text {eq }}<0.3 \mathrm{~mm}\right)$ & 1710833 & $674648(39.4)$ & $636655(37.2)$ & $1252175(73.2)$ \\
Large $\left(D_{\text {eq }} \geq 0.3 \mathrm{~mm}\right)$ & 1265670 & $34040(2.7)$ & $0(0.0)$ & $614144(48.5)$ \\
All particles & 5764722 & $3349750(58.1)$ & $2504337(43.4)$ & $4429756(76.8)$ \\
\hline
\end{tabular}

UWILD's liquid fraction for particles with $D_{\text {eq }}$ between 0.2 and $0.5 \mathrm{~mm}$ at temperatures between 0 and $5^{\circ} \mathrm{C}$ is approximately 0.5 (Fig. 8c). This is unrealistically low particularly for the warmer end of this temperature range, where most frozen particles would have melted. We note that UWILD, unlike Holroyd, achieves a liquid fraction near 1.0 for temperatures above $5^{\circ} \mathrm{C}$.

Holroyd also classifies many small particles $\left(D_{\mathrm{eq}}<\right.$ $0.1 \mathrm{~mm}$ ) as ice (Fig. 8e) at all temperatures. Its liquid fraction never exceeds 0.86 for small particles at temperatures above $-20^{\circ} \mathrm{C}$, whereas area ratio and UWILD have a liquid fraction near 1.0 (Fig. 8c and d). Holroyd's relatively low liquid fractions for small particles are unrealistic for temperatures above $0{ }^{\circ} \mathrm{C}$.

Area ratio classifies many large particles $\left(D_{\text {eq }}>0.3 \mathrm{~mm}\right)$ as liquid at low temperatures (Fig. 8d). Area ratio's liquid fraction rarely drops below 0.8 for particles with $D_{\text {eq }}>$ $0.2 \mathrm{~mm}$ and temperatures below $-5^{\circ} \mathrm{C}$, whereas Holroyd and UWILD have liquid fractions near 0.0 (Fig. 8c and e). While a liquid fraction between 0.5 and 0.8 for these particles is not physically impossible, the fact that there is no decrease in the liquid fraction with increasing particle size for particles with $D_{\text {eq }}$ between 0.5 and $1 \mathrm{~mm}$, where particle sizes and temperatures are inconsistent with supercooled drizzle formation, implies that area ratio's higher liquid fractions may be unrealistic.
There are also clear differences between the three algorithms' classifications in RH vs. particle size space (Fig. 8hj). UWILD and area ratio both have liquid fractions near 1.0 for small particles $\left(D_{\mathrm{eq}}<0.1 \mathrm{~mm}\right)$ near liquid saturation $(\mathrm{RH}=100 \%)$, whereas Holroyd has a liquid fraction closer to 0.75 for the same region. Uncertainty in $\mathrm{RH}$ is around $7 \%$, so while high liquid fractions are most common at liquid saturation, they occur at a wide range of RH values. Additionally, fluctuations in RH from dry-air entrainment and in-cloud circulation can lead to deviations from liquid saturation at $1 \mathrm{~Hz}$ resolution. UWILD classifies fewer particles as liquid in subsaturated air than either area ratio or Holroyd. In the mid-sized particle range $\left(0.1<D_{\mathrm{eq}}<0.2 \mathrm{~mm}\right)$, which includes drizzle, the liquid fraction is near 1.0 when the $\mathrm{RH}$ is close to $100 \%$, and it drops down to 0.2 when the $\mathrm{RH}$ decreases to $50 \%$.

Liquid particles can persist in subsaturated air at or below the cloud base, and these regions were purposefully sampled within the boundary layer during SOCRATES. Drizzle drops falling below liquid clouds evaporate in the subsaturated environment, reducing their size. Subsaturated air can also be associated with ice-dominated clouds, as $\mathrm{RH}_{\mathrm{i}}$ is higher than $\mathrm{RH}$ throughout the mixed-phase temperature range. In icedominated clouds, cloud droplets are produced at the turbulent cloud top and tend to freeze before forming drizzle drops. For both of these reasons, we expect the average size of liquid particles to decrease as the RH decreases below liq- 

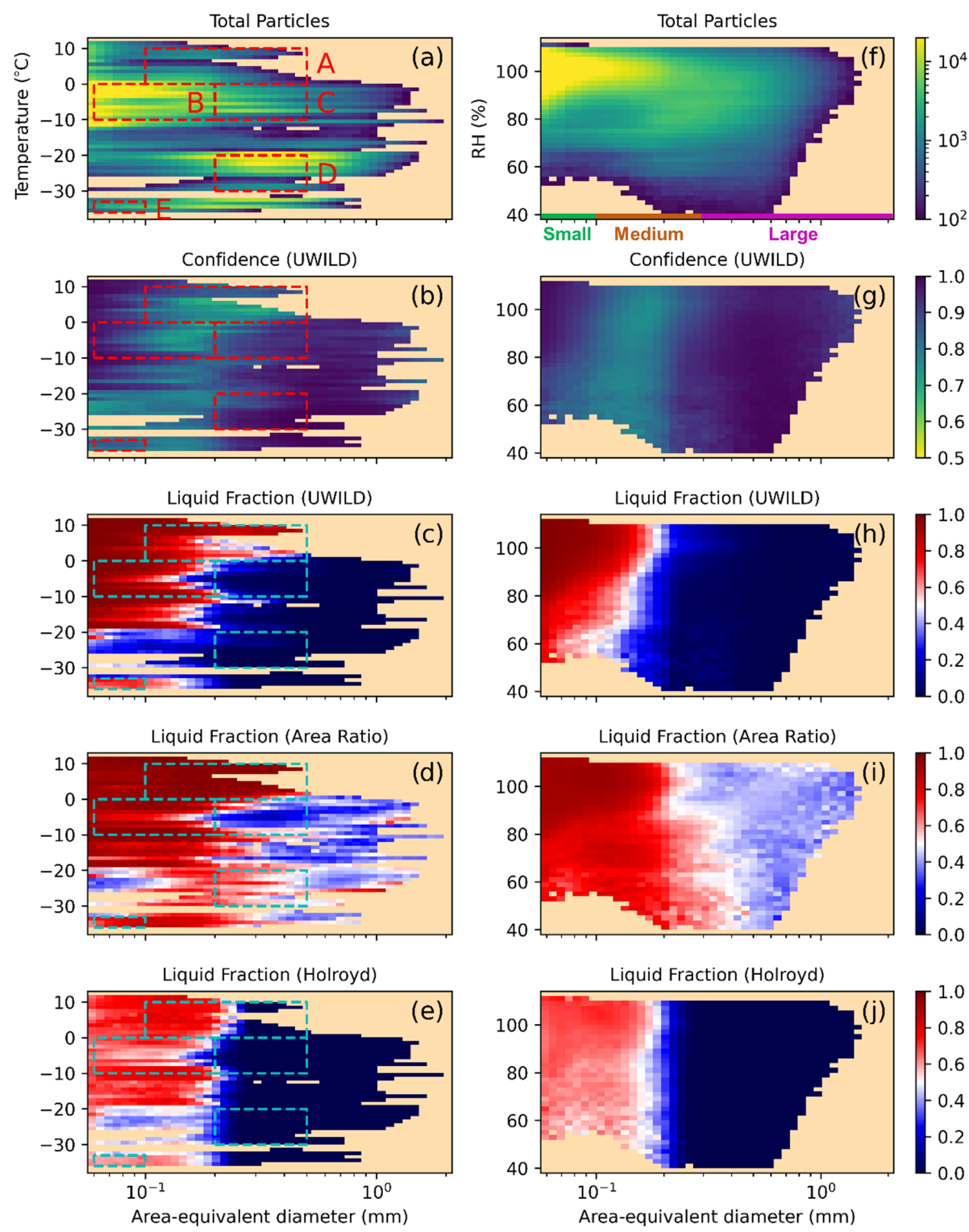

Figure 8. A 2D histogram of the number of particles meeting our criteria (row 1), UWILD confidence (row 2), and phase classifications for the three algorithms (rows 3-5) are shown in temperature-particle size phase space in the left column and relative-humidity-particlesize phase space in the right column. A threshold of 100 total particles per 2D histogram bin is used for both plots. SOCRATES flights RF01-RF14 are included.

uid saturation. UWILD is the only algorithm for which the $50 \%$ liquid fraction (in white) shifts to smaller sizes as RH decreases below $100 \%$. Thus, UWILD's lower liquid fractions in regions with $\mathrm{RH}<100 \%$, for particles in the midsized particle range $\left(0.1<D_{\mathrm{eq}}<0.2 \mathrm{~mm}\right)$, are more realistic than Holroyd's and area ratio's higher liquid fractions.

UWILD is the only algorithm of the three that can achieve liquid fractions near 0.0 and near 1.0, in both temperature vs. particle size space (Fig. 8c) and RH vs. particle size space (Fig. 8h). Thus, it has the flexibility to represent both (i) the liquid-only regions that we expect at the highest temperatures and near-liquid saturation and (ii) the ice-dominated regions that we expect at the lowest temperatures and the largest particles sizes, and in subsaturated regions.

The dashed boxes labeled a- $\mathrm{d}$ on the $2 \mathrm{D}$ histograms in the left column of Fig. 8 highlight areas of disagreement between the models, whereas box e highlights agreement between the models regarding the presence of supercooled water at $-35^{\circ} \mathrm{C}$. Figure 9 shows randomly sampled images from each of the five regions within the dashed boxes. Each particle image has the UWILD confidence displayed above the particle and the phase classifications from all three algorithms displayed below the particle. Since we have chosen to primarily focus on areas of disagreement between the al- 
gorithms, there are more misclassifications in these regions than in the dataset as a whole.

Box a highlights a region where area ratio has a liquid fraction near 1.0 across all size categories, Holroyd has a liquid fraction of about 0.75 for small particles and 0.0 for large particles, and UWILD has a liquid fraction of 1.0 for the highest temperatures and 0.5 for temperatures near $0^{\circ} \mathrm{C}$. Since temperature ranges from 0 to $10^{\circ} \mathrm{C}$ here, we expect that the particles are mainly liquid, although large ice particles can persist at temperatures above $0^{\circ} \mathrm{C}$. Furthermore, quasi-spherical frozen particles can have a close resemblance to large liquid drops, and the two cannot necessary be distinguished by eye from 2D-S images.

Randomly sampled particles from this region appear to be predominantly liquid due to the absence of rough edges along the perimeter and the high prevalence of quasi-spherical habits (Fig. 9a). Out of 50 randomly sampled images, area ratio classifies 1 particle as ice, Holroyd classifies 21 particles as ice, and UWILD classifies 12 particles as ice. Of the sampled particles classified by UWILD with a confidence $\geq 75 \%$, all but one of them appear to be properly identified as liquid. The only exception is one particle with a confidence of $81 \%$, which is likely misclassified due to being truncated at the end of the image buffer and having a lower area ratio as a result. A greater proportion of particles with a confidence below $75 \%$ are classified as ice by UWILD but are likely liquid, comprising about $55 \%$ of the sampled particles for these lower confidences. The misclassifications can be removed from UWILD, if desired, by filtering out particles that have a confidence of less than $75 \%$ and/or are touching the edge of the image buffer. Holroyd misclassifies nine more liquid particles as ice than UWILD but does not provide a measure of confidence that can be used to assess the likelihood of misclassification. Of the particles that UWILD likely misclassifies as ice, many have particularly large Poisson spots. Area is computed from shadowed diodes exclusively, so Poisson spots are not included. Additionally, particles with Poisson spots are resized following Korolev (2007). Both of these factors affect the calculation of area ratio and, thus, the phase classification of the particle.

Box $b$ highlights a temperature and size range where UWILD and area ratio are in agreement that the liquid fraction is near 1.0 but where Holroyd has a lower liquid fraction of about 0.75 . The 50 randomly sampled images shown in Fig. 9b appear to be mostly liquid with some irregular small ice crystals also present. UWILD performs better for these temperatures and particle size ranges than in Box a; it classifies four particles as ice, of which one is clearly liquid and three are unidentifiable by eye. Holroyd classifies 20 particles as ice, of which most are likely misclassifications.

Boxes $\mathrm{c}$ and $\mathrm{d}$ (Fig. 9c and d) both highlight regions where UWILD and Holroyd are in agreement that the liquid fraction is near 0.0 but where area ratio has a higher liquid fraction. The discrepancy is larger for box $d$, where area ratio has a liquid fraction of about 0.75 . Out of 45 randomly sampled images, area ratio misclassifies 8 quasi-spherical ice crystals as liquid for box c and at least 13 quasi-spherical ice crystals as liquid for box d. Particles in box c primarily come from boundary layer clouds, and most have columnar habits with small area ratios, although there are a smaller number of large quasi-spherical frozen particles with large area ratios present as well. Many of the particles in box $\mathrm{d}$ come from the atmospheric-river case described in Finlon et al. (2020) and, thus, are more likely to have quasi-spherical heavily rimed habits with large area ratios. The different particle habits explain the discrepancy in area ratio's performance in the two different regions.

In both boxes $\mathrm{c}$ and d, UWILD and Holroyd likely misclassify several large particles as ice, and those particles are largely associated with low confidence in UWILD. It is difficult to quantify this bias because there are several particles that could be either quasi-spherical frozen particles or large droplets and cannot be distinguished by eye. This does not mean that UWILD cannot classify those particles because it uses inter-arrival time in addition to image-derived parameters to make classifications.

Box e (Fig. 9e) highlights a region where all threephase discrimination algorithms have liquid fractions greater than 0.5 despite sampling very low temperatures $(-33$ to $\left.-36^{\circ} \mathrm{C}\right)$. Randomly sampled images with high confidence in UWILD have spherical habits, and most have Poisson spots as well, suggesting that the particles in this region are primarily liquid. UWILD and area ratio classify all 20 randomly sampled images as liquid, whereas Holroyd classifies 4 particles in the sample as ice. These particles are particularly small and lack Poisson spots, so we cannot identify their phase by eye. Nevertheless, it is clear that all three algorithms correctly identify this region as liquid-dominated. These particles were sampled during a period from RF03 that is plotted in the second half of Fig. 4e, where the aircraft skirted the top of an altostratus layer.

\section{Applications}

\subsection{Particle size distributions}

We use UWILD's classifications and confidences to compute median $1 \mathrm{~Hz}$ liquid and frozen particle size distributions (PSDs) and uncertainties for all SOCRATES data (14 flights). We show average PSDs for five different temperature ranges and for the whole dataset, in Fig. 10. The $x$ axis is $D_{\text {eq }}$ (consistent with other figures) and the $y$ axis is the particle concentration normalized by the common logarithm of the bin width. Both axes are plotted on a log scale. The dashed lines are deterministic distributions, which means they are generated using the UWILD classifications without taking the model confidence into account. All classified particles are used for this analysis regardless of model confidence. The solid lines and shaded areas around them are the 


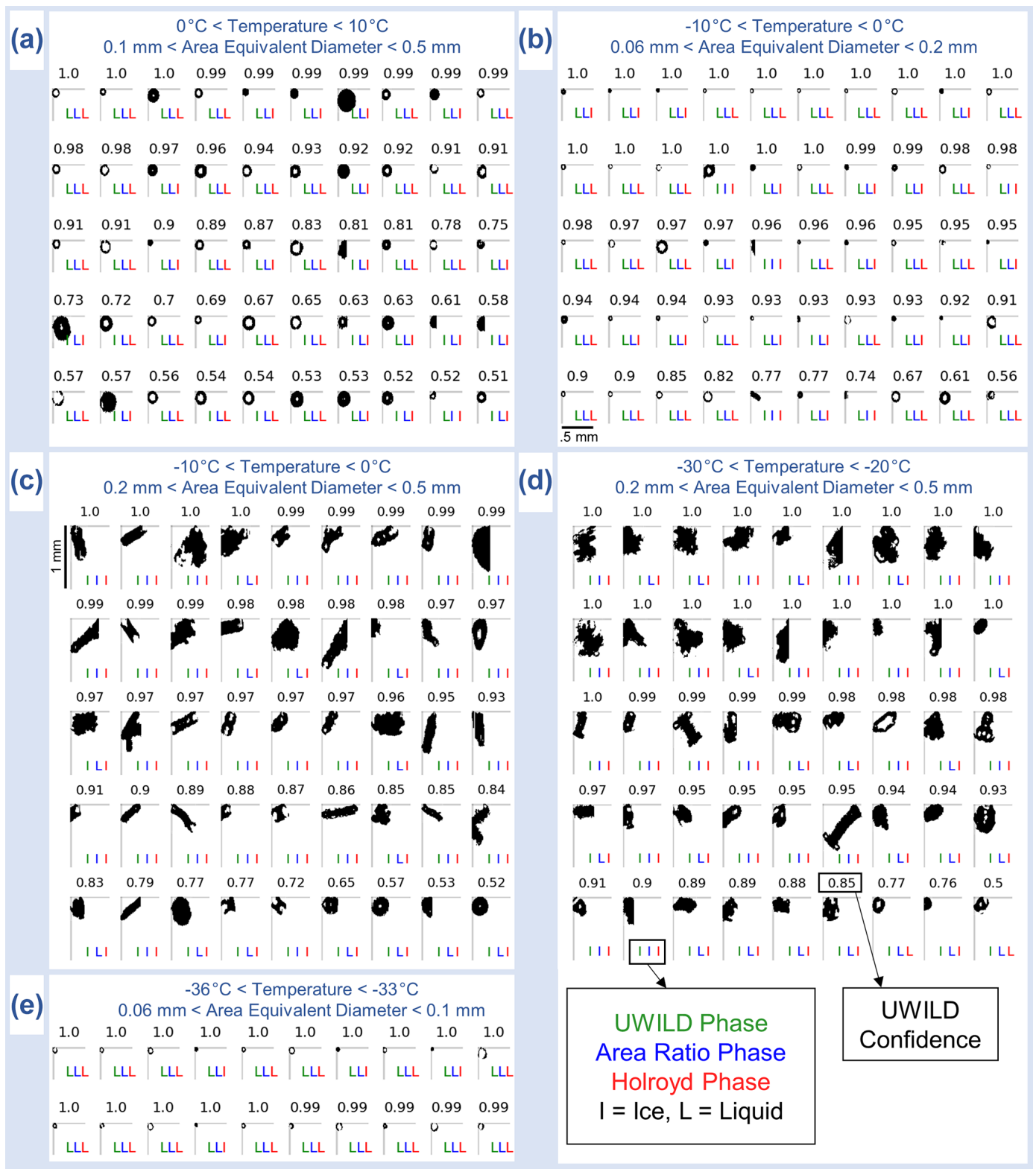

Figure 9. Randomly sampled images are shown for the five regions overlaid on the temperature-particle-size phase space in Fig. 8. UWILD confidence is displayed above each particle, and, from left to right, UWILD phase (green), area ratio phase (blue), and Holroyd phase (red) are displayed below each particle. The time dimension is vertical, and the photodiode dimension is horizontal.

median and interquartile range of 30 bootstrapped samples which are generated using the model confidence. For example, if a particle is classified as ice with $75 \%$ confidence then it is considered an ice particle for the deterministic distribution, but, on average, it will be considered an ice (liquid) particle in $75 \%(25 \%)$ of the bootstrapped samples. Note that due to the log scale on the $y$ axis, the effect of bootstrapping is mainly noticeable where concentrations are small and is strongest where the differences between ice and liquid concentrations span orders of magnitude. Note also that the bootstrapped distributions fall entirely between the determinis- tic distributions. This can be understood by considering, for example, the smallest particles just below $0{ }^{\circ} \mathrm{C}$, where there are approximately 100 times as many liquid particles as ice. If, when taking into consideration model confidence, $2 \%$ of the liquid particles are reclassified as ice, this is barely noticeable in the liquid particle concentration but results in a tripling of the ice particle concentration (Fig. 10c). The bootstrapped PSDs are better representations of the true PSDs, so considering model confidence is most essential in the areas in Fig. 10 where there are large discrepancies between the 
deterministic and bootstrapped distributions (e.g., estimating sub-millimeter ice particle concentrations around $0^{\circ} \mathrm{C}$ ).

Within the SOCRATES dataset, small particles $\left(D_{\mathrm{eq}}<\right.$ $0.1 \mathrm{~mm}$ ) are more likely to be liquid at all temperature ranges but have much higher concentrations and are more liquid-dominated above $-20^{\circ} \mathrm{C}$ (Fig. 10b-e). The concentrations of medium-sized $\left(0.1 \mathrm{~mm}<D_{\text {eq }}<0.3 \mathrm{~mm}\right)$ and large $\left(D_{\text {eq }}>0.3 \mathrm{~mm}\right)$ particles decrease as temperature increases. Medium-sized particles are ice-dominated between -40 and $-20^{\circ} \mathrm{C}$ (Fig. 10a) and liquid-dominated above $-5^{\circ} \mathrm{C}$ (Fig. 10c-e). Large particles are liquid-dominated above $5^{\circ} \mathrm{C}$ (Fig. 10e), where drizzle formation becomes the dominant mode of precipitation. The largest particles $\left(D_{\text {eq }}>1 \mathrm{~mm}\right)$ are ice-dominated at all temperatures but have small concentrations $\left(<2 \times 10^{-4} \mathrm{~cm}^{-3}\right)$ above $5{ }^{\circ} \mathrm{C}$ (Fig. 10e). The crossover point, or the $D_{\text {eq }}$ at which the PSDs transition from being liquid-dominated to being icedominated, is $0.1 \mathrm{~mm}$ for temperatures between -40 and $-20^{\circ} \mathrm{C}$ (Fig. 10a), 0.17-0.33 $\mathrm{mm}$ for temperatures between -20 and $5^{\circ} \mathrm{C}$ (Fig. 10b-d) and $0.7 \mathrm{~mm}$ at higher temperatures (Fig. 10e). The crossover point for the whole dataset is $0.17 \mathrm{~mm}$ (Fig. 10f), which is in agreement with the phase classifications from the PHIPS for all SOCRATES flights (Waitz et al., 2021, figure 8). Small ice crystals and large liquid drops are associated with the most uncertainty at all temperature ranges.

In Sect. 4, we showed that UWILD misclassifies some large liquid particles as ice. We examined 200 randomly sampled images of particles with $D_{\text {eq }}>0.16 \mathrm{~mm}$ from temperatures between 0 and $5^{\circ} \mathrm{C}$ and found that $16 \%$ of particles classified as ice are actually liquid (not shown). These misclassified particles universally lack Poisson spots. Thus, large particles within that temperature range are indeed icedominated but to a lesser extent than what the PSDs suggest. We also examined 200 randomly sampled 2D-S images of particles with $D_{\text {eq }}>1 \mathrm{~mm}$ from temperatures between 5 and $40^{\circ} \mathrm{C}$ and found that all particles are classified as ice but are actually liquid (not shown). These particles have an elongated shape due to being distorted by the instrument inlet, lack a Poisson spot, and often touch the edge of the image buffer so that they are partially cut off. We note that classification skill decreases in general for particles approaching $1 \mathrm{~mm}$ because particles of this size are less likely to be fully imaged by the instrument. Thus, we caution that phase classifications for the largest particles may be less certain and that the $5-40{ }^{\circ} \mathrm{C}$ temperature range is particularly affected by misclassifications of large drops as ice. We note that there are so few of these large misclassified liquid particles at high temperatures that they do not show up in Fig. 8c, which uses a threshold of 100 particles for each 2D histogram bin.

\subsection{Cloud phase heterogeneity}

We also use UWILD's classifications and confidences to compute a $1 \mathrm{~Hz}$ estimate of sub-1 Hz cloud phase hetero- geneity. Cloud phase heterogeneity, or the degree to which liquid and ice particles are evenly mixed within mixed-phase clouds, can influence cloud radiative (Sun and Shine, 1994) and thermodynamic properties (Korolev et al., 2017). It may also modulate the rates of certain mixed-phase processes such as the Wegener-Bergeron-Findeisen process (Tan and Storelvmo, 2016) and has implications for how those processes should be parameterized in microphysics models. Most studies of cloud phase heterogeneity from in situ observations have computed heterogeneity metrics using $1 \mathrm{~Hz}$ phase data (Cober et al., 2001a; Korolev et al., 2003; Field et al., 2004; D’Alessandro et al., 2019, 2021). Field et al. (2004) used PSDs from the Small Ice Detector in combination with other in situ measurements to identify cloud phase and found that segments as short as $100 \mathrm{~m}$ could contain both liquid and ice. They used $1 \mathrm{~Hz}$ data but could investigate relatively small length scales due to low aircraft speeds (100$120 \mathrm{~m} \mathrm{~s}^{-1}$ ). Here, we use single-particle phase classifications to investigate sub- $1 \mathrm{~Hz}$ cloud phase heterogeneity, and we identify mixed-phase periods on the meter-scale.

We derive an estimate of sub- $1 \mathrm{~Hz}$ heterogeneity by considering whether adjacent particles in the 2D-S image buffer are of the same phase or of different phases, which we denote as a phase "flip" (from ice to liquid or from liquid to ice). If, for a $1 \mathrm{~s}$ period, there are many phase flips given the number of particles, that sample is more heterogeneous than one where there are few (or no) phase flips for a population of particles. We leverage the fact that our classifications are probabilistic in determining phase flips and create a probabilistic phase flip prediction as well. Given two adjacent particles $p_{1}$ and $p_{2}$,

$$
\begin{aligned}
P(\text { flip }) & =P\left(p_{1}=\text { ice }\right) \times P\left(p_{2}=\text { liquid }\right) \\
& +P\left(p_{1}=\text { liquid }\right) \times P\left(p_{2}=\text { ice }\right) .
\end{aligned}
$$

We estimate the most likely number of flips over all particles within a given sample by adding these probabilities together. Thus a hypothetical sample containing 100 particles may have between 0 flips (completely homogeneous sample with $100 \%$ classification confidence on all particles) and 99 flips (particles are alternating $100 \%$ likely ice and $100 \%$ likely liquid), although both of these extremes are unlikely with our probabilistic estimate. A limitation of this heterogeneity estimate is that it implicitly assumes that phase flip probabilities are independent. An advantage of this metric is that it avoids using particle mass to compute phase heterogeneity. Ice particle mass estimated from 2D-S images can vary over an order of magnitude depending on the assumed mass-dimensional relationship (Wu et al., 2019), and more reliable measurements from a Nevzorov instrument with a deep cone (Korolev et al., 2003) were not available during SOCRATES.

We create a $1 \mathrm{~Hz}$ heterogeneity measure, which we refer to as the phase flip fraction, by dividing the number of probabilistic phase flips described above by the total number of 

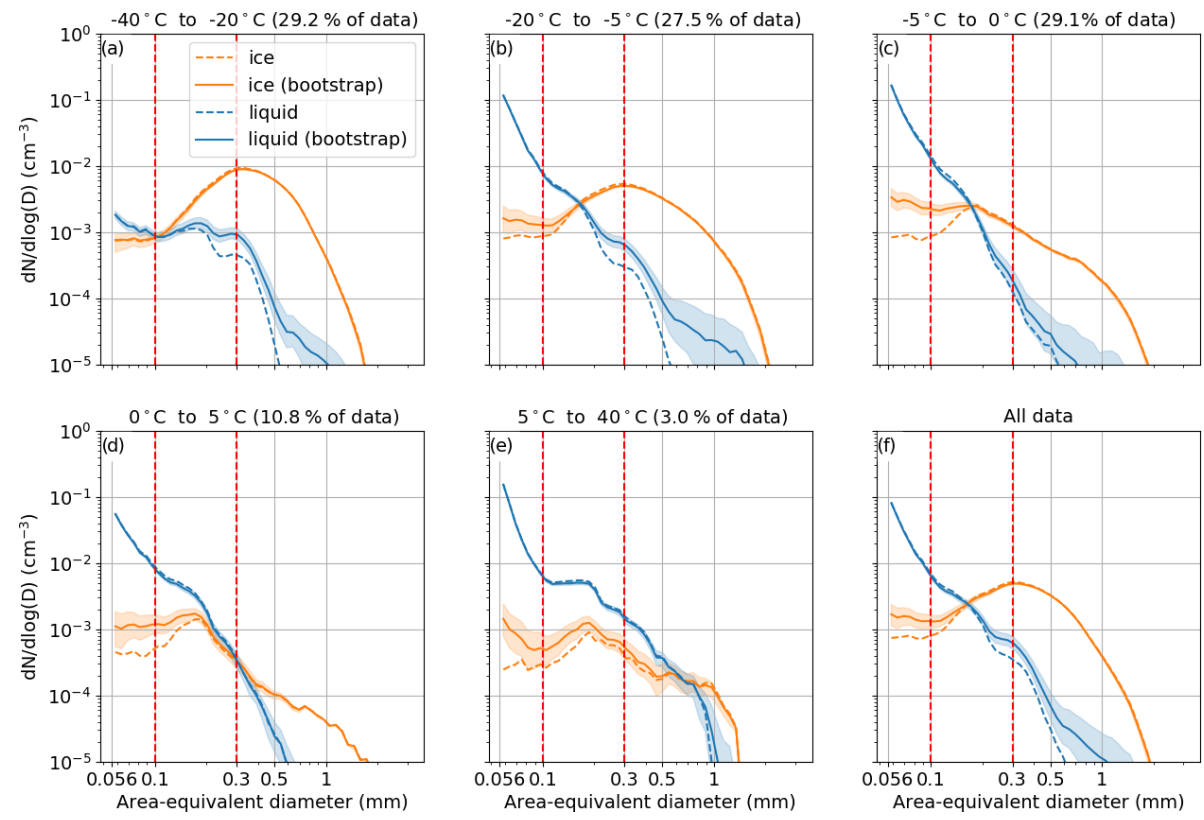

Figure 10. Particle size distributions are averaged over $1 \mathrm{~Hz}$ data from SOCRATES flights RF01-RF14 and shown for six different temperature ranges. Dashed lines are deterministic predictions from UWILD. Solid lines and shaded areas are medians and interquartile ranges, respectively, for 30 bootstrapped samples generated from UWILD confidences. Red dashed lines separate the three different size classes.

particles imaged by the 2D-S within $1 \mathrm{~s}$. We implicitly assume that all unclassified particles, which are mainly particles with fewer than 25 pixels and $D_{\text {eq }}<0.056 \mathrm{~mm}$, are liquid, which is a reasonable extrapolation from our PSDs (Fig. 10f), as only $1 \%$ of the smallest particles are classified as ice. However, this assumption may lead to an underestimate in phase flips for the coldest samples $\left(-40^{\circ} \mathrm{C}<T<\right.$ $-20^{\circ} \mathrm{C}$ ), where there are similar numbers of small droplets and small ice crystals (Fig. 10a).

Figure 11 shows a 2D histogram with phase flips on the $x$ axis and the total number of 2D-S imaged particles on the $y$ axis, with white lines indicating lines of constant phase flip fraction. We see two distinct modes in this phase space: $1 \mathrm{~s}$ samples with total particle counts below 1000 typically have between 0.02 and 0.5 flips per particle, whereas $1 \mathrm{~s} \mathrm{sam-}$ ples with total particle counts between 3000 and 30000 typically have between 0.002 and 0.0005 flips per particle. In the SOCRATES dataset, high total particle counts generally indicate the presence of many small droplets. Since these samples are dominated in number by the liquid phase, they have low phase heterogeneity.

Figure 12 shows two examples of meter-scale phase heterogeneity. The statistics of the $1 \mathrm{~s}$ periods containing the plotted segments are included to the right of the image strips. Grey lines bound each particle, and all particles that are large enough to be classified and are not suspected artifacts are labeled with their UWILD classifications and the model confidence in parentheses. Red labels are used for ice classifications with confidence $\geq 75 \%$; blue labels for liquid classifi-
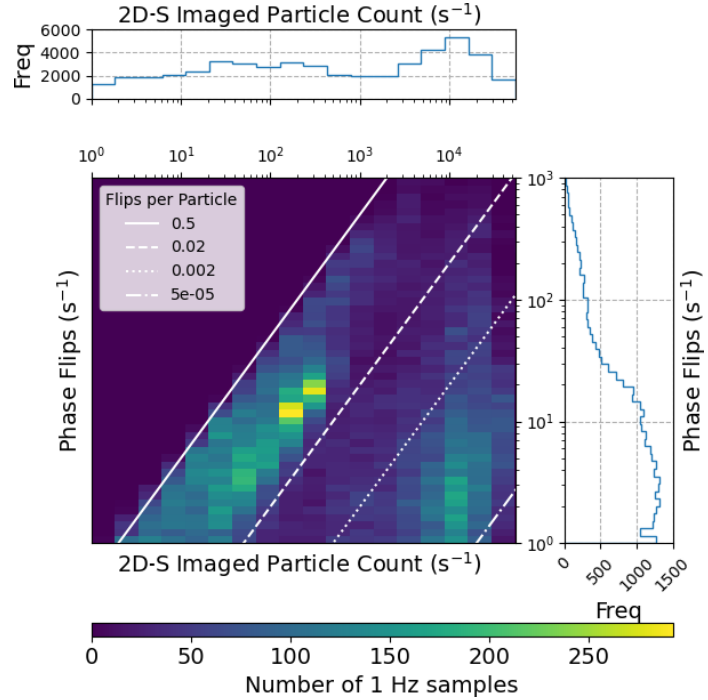

Figure 11. A 2D histogram of $1 \mathrm{~Hz}$ particle phase flips ( $y$ axis) and $1 \mathrm{~Hz}$ total particles (including both successfully classified particles and unclassifiable particles) is shown in the large plot. A 1D histogram of $1 \mathrm{~Hz}$ total particles is shown on top, on a shared $x$ axis, and a $1 \mathrm{D}$ histogram of $1 \mathrm{~Hz}$ phase flips per second is shown on the right, on a shared $y$ axis. A value closer to the upper left of the plot indicates a higher degree of particle heterogeneity. White lines indicate lines of constant heterogeneity for varying particle counts. SOCRATES flights RF01-RF14 are included. 
Examples of meter-scale phase heterogeneity during SOCRATES

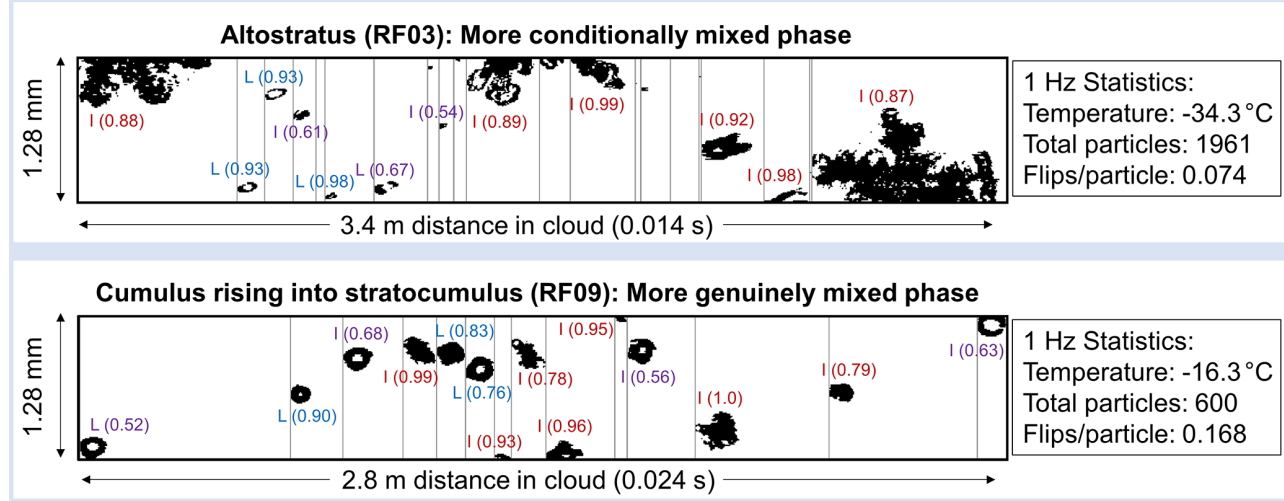

Figure 12. Short periods from two different cloud types highlight instances of meter-scale heterogeneity in the SOCRATES dataset. The photodiode dimension is vertical, and the time dimension is horizontal, which is the reverse of Fig. 9. Particle labels show UWILD classifications and UWILD confidences in parentheses. Red indicates ice classifications with confidences greater than $75 \%$; blue indicates liquid classifications with confidences greater than $75 \%$; and purple indicates ice or liquid classifications with confidences less than $75 \%$

cations with confidence $\geq 75 \%$; and purple labels for ice or liquid classifications with confidence $<75 \%$.

In the example in the first row, there is a pocket of small droplets surrounded by large ice crystals within a $3.4 \mathrm{~m}$ segment of cloud. There are three particles within the pocket that have low model confidence and thus may be small droplets or small ice crystals. This example resembles the conditionally mixed-phase condition described by Korolev et al. (2017), where the cloud is single phase if you look at a small enough length scale. For the segment of cloud shown, that length scale is approximately $1 \mathrm{~m}$. The second row shows alternating liquid and ice particles of similar size within a $2.8 \mathrm{~m}$ segment of cloud. Here, there is a larger proportion of lowconfidence particles that could be either liquid or ice. This is a result of UWILD's tendency, described in Sect. 4, to classify some large liquid drops as ice. There is a sequence of four particles classified as $\mathrm{I}-\mathrm{L}-\mathrm{L}-\mathrm{I}$, all with high confidence, towards the center of the image strip. This example more closely resembles the genuinely mixed-phase condition described by Korolev et al. (2017) because the phase changes every one or two particles. The difference in the heterogeneity between the two regions is captured in the $1 \mathrm{~s}$ flips per particle, which is about twice as high for the period in the bottom row than it is for the period in the top row. However, there may be fewer or greater flips per particle in the periods shown than in the $1 \mathrm{~s}$ periods if the periods shown are not representative of the entire $1 \mathrm{~s}$ periods.

\section{Conclusions}

In situ observations of Southern Ocean cloud phases, vital for evaluating simulations and remote sensing products, were sparse prior to the Southern Ocean Clouds, Radiation, Aerosol Transport Experimental Study (SOCRATES) cam- paign in January-February of 2018. The SOCRATES dataset includes nearly 6 million Two-Dimensional Stereo (2D-S) shadow images of particles with 25 or more pixels and with area equivalent diameters $\left(D_{\text {eq }}\right)$ greater than $0.056 \mathrm{~mm}$, which are good candidates for single-particle phase classification. Here, we introduce the University of Washington Ice-Liquid Discriminator (UWILD), a phase classification algorithm that takes a random forest approach, and show that it outperforms two existing phase classification algorithms which have been applied to 2D-S images from SOCRATES. In particular, UWILD has the flexibility to identify both liquid-dominated and ice-dominated regions in the dataset, whereas the other two algorithms both demonstrate strong biases in favor of one phase. UWILD also returns a model confidence for each classification, which is invaluable for computing uncertainties on variables derived from its classifications and for filtering out particles that have a higher likelihood of being misclassified. We believe that the performance of UWILD is limited largely by the SOCRATES 2D-S data quality (many particle images are out of focus) and the challenges of building a train-test-validation (TTV) set and not the choice of machine learning model. Thus, we would expect to see only modest gains in performance from employing more sophisticated machine learning models.

Since many hydrometeors within mixed-phase clouds are unidentifiable by eye, we use in situ observations of atmospheric parameters to select liquid-only and ice-dominated periods within SOCRATES to build a TTV set. If we had limited the TTV set to particles identifiable by eye, as most studies applying machine learning to airborne probe images have done, the TTV set would be less representative of the SOCRATES dataset. UWILD's most prominent bias is the misclassification of larger liquid particles $\left(D_{\text {eq }}>0.2 \mathrm{~mm}\right)$ as ice. A small percentage $(\sim 0.6 \%)$ of particles in the icedominated training data are likely liquid, and this may con- 
tribute to this bias. UWILD's lower skill in distinguishing larger liquid drops from ice particles is shared by Holroyd and by the automated Particle Habit Imaging and Polar Scattering (PHIPS) algorithm which uses scattering phase functions to identify particle sphericity. Thus, we posit that distinguishing drizzle droplets and raindrops from ice crystals is a major outstanding problem in hydrometeor phase discrimination within mixed-phase clouds. We also note that larger liquid drops that are misclassified by UWILD typically have model confidence below $75 \%$ or are touching the edge of the image buffer and can be easily filtered out of the dataset if desired.

We use classifications and confidence from UWILD to generate particle size distributions (PSDs) for the whole SOCRATES dataset and find that hydrometeors with $D_{\text {eq }}$ greater than $0.15 \mathrm{~mm}$ are ice-dominated and that smaller particles are liquid-dominated, which is in agreement with phase classifications from the PHIPS for the SOCRATES dataset. We also develop a novel estimate of sub- $1 \mathrm{~Hz}$ phase heterogeneity by tallying the number of probabilistic phase flips per particle within $1 \mathrm{~s}$ periods, which we refer to as the phase flip fraction. This particle-number-based approach to estimating heterogeneity avoids uncertainties in estimating particle mass. We use the phase flip fraction to identify two periods in the SOCRATES dataset exhibiting meter-scale phase heterogeneity.

The SOCRATES campaign sampled mixed-phase Southern Ocean clouds with the goal of improving their representation in climate models. Here, we process SOCRATES in situ observations from the 2D-S to create datasets that can be more effectively compared with atmospheric models and remote sensing datasets. Liquid and ice PSDs can be used to evaluate microphysical model output. Single-particle phase classifications can be coarsened and compared with cloud top phase products from Himawari and MODIS. Singleparticle classifications from UWILD, as well as the $1 \mathrm{~Hz}$ variables that we derive from them, are also useful for informing the development of microphysics parameterizations. Large droplets are necessary for Hallett-Mossop rime splintering and droplet freezing, and estimating their concentrations from liquid classifications of 2D-S images is useful for determining an upper bound on the rate of these ice production processes. Additionally, mixed-phase processes such as the Wegener-Bergeron-Findeisen mechanism for rapid ice growth and Hallett-Mossop rime splintering may operate more slowly in conditionally mixed-phase clouds, which have a small number of flips per particle, than genuinely mixed-phase clouds, which have a large number of flips per particle. UWILD is publicly available, and we encourage readers to adapt it for other in situ datasets to examine processes controlling phase partitioning in mixed-phase clouds globally.

\section{Appendix A: Particle features used for phase classification}

Throughout this section, we use the term "particle property" to refer to any quantity that is computed from the 2D-S dataset. We use the term "particle feature" to refer to particle properties that we input into UWILD. Figure A1 shows how particle features are computed from 2D-S images. Particle properties that can be represented visually are shown in the top two boxes, using two example particles. Particle properties in the bottom box are functions of the particle properties shown in the top two boxes and cannot directly be visualized. All of the particle properties shown in Fig. A1 are particle features except "smallest bounding circle", which is used to compute max_dimension and area_ratio, and "perimeter", which is used to compute fine_detail_ratio. Underscores are left out of the particle names in Fig. A1.

We show histograms of 14 particle features for the TTV set and the 14 SOCRATES flights analyzed in this study, in Fig. A2. We do not include the parameter touching_edge in Figs. A1 or A2 because it is binary. The histograms are plotted on a $\log$ scale so that the tails of their distributions are visible. About $0.6 \%$ of particle images from the SOCRATES dataset that we analyzed here are out of sample. This means that at least one particle parameter has a value that is outside of the range of the TTV set. Such particles are usually out of sample because they are larger than all of the particles in the TTV set. We examined 45 randomly sampled images from the SOCRATES dataset that have an area-equivalent diameter $\left(D_{\text {eq }}\right)$ greater than all particles in the TTV set. These particles are mainly heavily rimed aggregates. All of them are clearly frozen, and UWILD classifies them as such. Thus, we do not believe that the small percentage of out-of-sample particles present in the SOCRATES dataset reduces the performance of UWILD.

Area ratio is greater than 1.0 about $25 \%$ of the time. This is because a correction is applied to the calculation of the maximum dimension following Korolev (2007) when the diode at the center of the minimum circle bounding the particle is unshadowed, which is the case for particles featuring Poisson spots. In these cases, the area of a circle with a diameter equal to the corrected maximum dimension can be smaller than the particle area. 


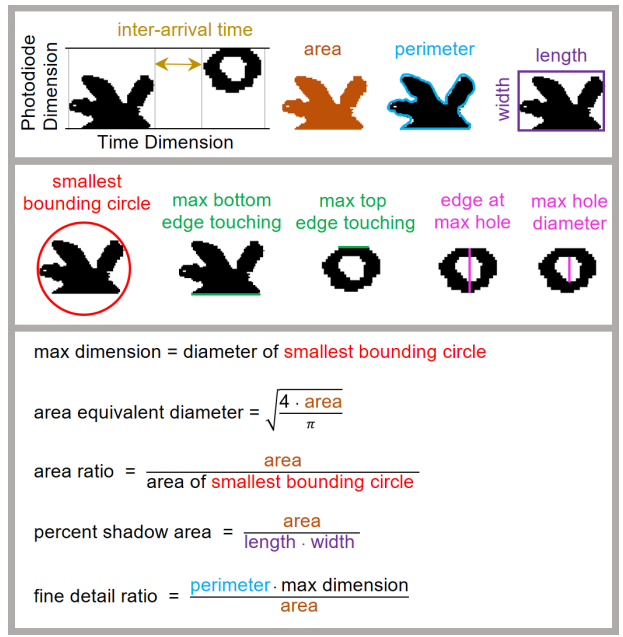

Figure A1. Schematic showing how to compute particle features from 2D-S particle images. Particle features are listed in Table 1. Underscores are left out of the feature names. touching_edge is not shown here because it is a binary variable.
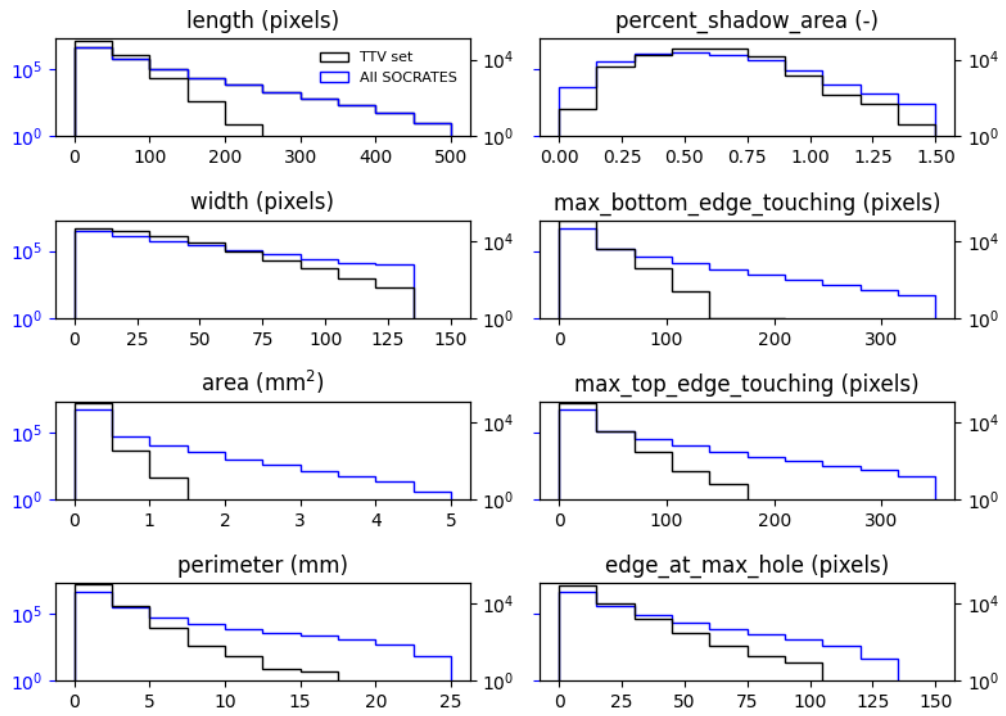

edge_at_max_hole (pixels)
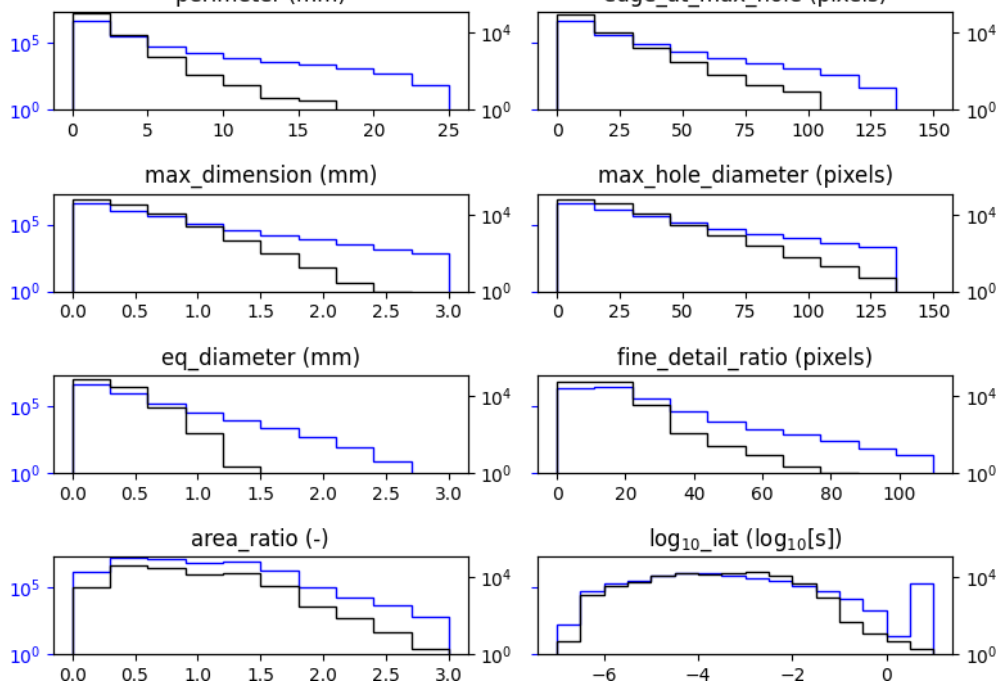

Figure A2. Histograms of particle features are shown for the TTV set (black) and for SOCRATES flights RF01-RF14 (blue), which are analyzed in Sects. 4 and 5. touching_edge is not shown here because it is a binary variable. 
Code and data availability. The 2D-S (https://data.eol.ucar.edu/ dataset/552.009, EOL Data Support, 2020), VCSEL (https:// doi.org/10.26023/V925-2H41-SD0F, Diao, 2021), PHIPS (https: //doi.org/10.5065/D62B8WWF, Schnaiter, 2018a; https://doi.org/ 10.5065/D6639NKQ, Schnaiter, 2018b), and aircraft (https://doi. org/10.5065/D6M32TM9, EOL, 2019) data used in this study are found on the NCAR EOL data archive. The software packages used to process the OAP data (https://doi.org/10.5281/zenodo.1285969, McFarquhar et al., 2018) and to run the random forest model (https://doi.org/10.5281/zenodo.5197777, Mohrmann et al., 2021b) are publicly available as GitHub repositories (https: //github.com/joefinlon/UIOPS/tree/v3.1.4 and https:/github.com/ $\mathrm{jkcm} / \mathrm{UW}$-particle-phase-classifier/tree/v1.1, last access: 1 November 2021). The data containing $1 \mathrm{~Hz}$ phase-partitioned PSDs and phase flip fraction estimates are publicly available on the NCAR EOL data archive (https://doi.org/10.26023/PA5W-4DRX-W50A, Mohrmann et al., 2021a).

Author contributions. RA prepared the manuscript with contributions from all co-authors and led the analysis of the phase classifications from the three different algorithms for the whole SOCRATES dataset (Sect. 4). JM led the development of UWILD, with contributions from $\mathrm{JL}$ and $\mathrm{IH}$, and computed size distributions and phase heterogeneity metrics from UWILD's classifications and model confidences (Sect. 5). JF processed the 2D-S data to obtain particle features used in the machine learning model and contributed expertise on the 2D-S instrument and the SOCRATES campaign. JL and IH trained and tuned UWILD and evaluated its performance on the test set (Sect. 4). In particular, JL computed $F_{1}$ scores for different particle categories, and IH computed permutation feature importance. RW provided continuous feedback and guidance during the study. MD recalibrated the water vapor data from the VCSEL instrument and provided comments for the manuscript.

Competing interests. The contact author has declared that neither they nor their co-authors have any competing interests.

Disclaimer. Publisher's note: Copernicus Publications remains neutral with regard to jurisdictional claims in published maps and institutional affiliations.

Acknowledgements. The authors acknowledge all SOCRATES scientists for collecting the data used in this study. The authors are grateful to Emma Järvinen and Fritz Waitz for their help with interpreting the PHIPS data, Wei Wu for their work on the initial 2D-S single-particle phase classification, and Greg McFarquhar for their discussion of the SOCRATES microphysics measurements. Minghui Diao acknowledges support from the National Center for Atmospheric Research (NCAR) Advanced Study Program (ASP) Faculty Fellowship in 2018.

Financial support. This research has been supported by the National Science Foundation (grant nos. AGS-1660609 and OPP1744965).
Review statement. This paper was edited by Hartmut Herrmann and reviewed by Darrel Baumgardner and Annika Lauber.

\section{References}

Abdelmonem, A., Järvinen, E., Duft, D., Hirst, E., Vogt, S., Leisner, T., and Schnaiter, M.: PHIPS-HALO: the airborne Particle Habit Imaging and Polar Scattering probe - Part 1: Design and operation, Atmos. Meas. Tech., 9, 3131-3144, https://doi.org/10.5194/amt-9-3131-2016, 2016.

Albrecht, B. A.: Aerosols, Cloud Microphysics, and Fractional Cloudiness, Science, 245, 1227-1230, https://doi.org/10.1126/science.245.4923.1227, 1989.

Arago, F. and Gay-Lussac, J.: Annales de chimie et de physique, Chez Crochard, available at: https://books.google.com/books? id=BspOAQAAMAAJ (last access: 19 January 2021), 1819.

Baumgardner, D. and Korolev, A.: Airspeed Corrections for Optical Array Probe Sample Volumes, J. Atmos. Ocean. Tech., 14, 1224-1229, https://doi.org/10.1175/15200426(1997)014<1224:acfoap>2.0.co;2, 1997.

Baumgardner, D., Abel, S. J., Axisa, D., Cotton, R., Crosier, J., Field, P., Gurganus, C., Heymsfield, A., Korolev, A., Krämer, M., Lawson, P., McFarquhar, G., Ulanowski, Z., and Um, J.: Cloud Ice Properties: In Situ Measurement Challenges, Meteor. Mon., 58, 9.1-9.23, https://doi.org/10.1175/amsmonographs-d16-0011.1, 2017.

Bergeron, T.: Über die dreidimensional verknüpfende Wetteranalyse, Geophys. Norv., 5, 1-111, 1928.

Bjordal, J., Storelvmo, T., Alterskjær, K., and Carlsen, T.: Equilibrium climate sensitivity above $5{ }^{\circ} \mathrm{C}$ plausible due to state-dependent cloud feedback, Nat. Geosci., 13, 718-721, https://doi.org/10.1038/s41561-020-00649-1, 2020.

Bodas-Salcedo, A., Hill, P. G., Furtado, K., Williams, K. D., Field, P. R., Manners, J. C., Hyder, P., and Kato, S.: Large contribution of supercooled liquid clouds to the solar radiation budget of the Southern Ocean, J. Climate, 29, 4213-4228, https://doi.org/10.1175/jcli-d-15-0564.1, 2016.

Bower, K. N., Moss, S. J., Johnson, D. W., Choularton, T. W., Latham, J., Brown, P. R. A., Blyth, A. M., and Cardwell, J.: A parametrization of the ice water content observed in frontal and convective clouds, Q. J. Roy. Meteor. Soc., 122, 1815-1844, https://doi.org/10.1002/qj.49712253605, 1996.

Breiman, L.: Random forests, Mach. Learn., 45, 5-32, https://doi.org/10.1023/a:1010933404324, 2001.

Cober, S. G., Isaac, G. A., Korolev, A. V., and Strapp, J. W.: Assessing cloud-phase conditions, J. Appl. Meteorol., 40, 1967-1983, https://doi.org/10.1175/15200450(2001)040<1967:acpc>2.0.co;2, 2001a.

Cober, S. G., Isaac, G. A., and Strapp, J. W.: Characterizations of Aircraft Icing Environments that Include Supercooled Large Drops, J. Appl. Meteorol., 40, 1984-2002, https://doi.org/10.1175/15200450(2001)040<1984:coaiet>2.0.co;2, 2001b.

Czys, R. R. and Schoen Petersen, M.: A RoughnessDetection Technique for Objectively Classifying Drops and Graupel in 2D-Image Records, J. Atmos. Ocean. Tech., 9, 242-257, https://doi.org/10.1175/15200426(1992)009<0242:ardtfo>2.0.co;2, 1992. 
D'Alessandro, J. J., McFarquhar, G. M., Wu, W., Stith, J. L., Jensen, J. B., and Rauber, R. M.: Characterizing the Occurrence and Spatial Heterogeneity of Liquid, Ice, and Mixed Phase LowLevel Clouds Over the Southern Ocean Using in Situ Observations Acquired During SOCRATES, J. Geophys. Res.-Atmos., 126, e2020JD034482, https://doi.org/10.1029/2020JD034482, 2021.

Diao, M.: VCSEL $25 \mathrm{~Hz}$ Water Vapor Data, Version 2.0, EOL data [data set], https://doi.org/10.26023/V925-2H41-SD0F, 2021.

D’Alessandro, J. J., Diao, M., Wu, C., Liu, X., Jensen, J. B., and Stephens, B. B.: Cloud Phase and Relative Humidity Distributions over the Southern Ocean in Austral Summer Based on In Situ Observations and CAM5 Simulations, J. Climate, 32, 27812805, https://doi.org/10.1175/jcli-d-18-0232.1, 2019.

EOL: Low Rate (LRT - 1 sps) Navigation, State Parameter, and Microphysics Flight-Level Data, Version 1.3, EOL data [data set], https://doi.org/10.5065/D6M32TM9, 2019.

EOL Data Support: NSF/NCAR GV HIAPER Raw 2D-S Imagery, EOL data [data set], available at: https://data.eol.ucar.edu/ dataset/552.009, last access: 7 August 2020.

Field, P. R.: Aircraft observations of ice crystal evolution in an altostratus cloud, J. Atmos. Sci., 56, 1925-1941, https://doi.org/10.1175/15200469(1999)056<1925:aooice>2.0.co;2, 1999.

Field, P. R. and Heymsfield, A. J.: Importance of snow to global precipitation, Geophys. Res. Lett., 42, 9512-9520, https://doi.org/10.1002/2015GL065497, 2015.

Field, P. R., Hogan, R. J., Brown, P. R. A., Illingworth, A. J., Choularton, T. W., Kaye, P. H., Hirst, E., and Greenaway, R.: Simultaneous radar and aircraft observations of mixed-phase cloud at the 100m scale, Q. J. Roy. Meteor. Soc., 130, 1877-1904, https://doi.org/10.1256/qj.03.102, 2004.

Finlon, J. A., Rauber, R. M., Wu, W., Zaremba, T. J., McFarquhar, G. M., Nesbitt, S. W., Schnaiter, M., Jarvinen, E., Waitz, F., Hill, T. C. J., and DeMott, P. J.: Structure of an Atmospheric River Over Australia and the Southern Ocean: I I. Microphysical Evolution, J. Geophys. Res.-Atmos., 125, e2020JD032514, https://doi.org/10.1029/2020jd032514, 2020.

Heymsfield, A. J. and Baumgardner, D.: Summary of a Workshop on Processing 2-D Probe Data, B. Am. Meteorol. Soc., 66, 437440, https://doi.org/10.1175/1520-0477-66.4.437, 1985.

Heymsfield, A. J. and Parrish, J. L.: A Computational Technique for Increasing the Effective Sampling Volume of the PMS Two-Dimensional Particle Size Spectrometer, J. Appl. Meteorol. Clim., 17, 1566-1572, https://doi.org/10.1175/15200450(1978)017<1566:actfit>2.0.co;2, 1978.

Holroyd, E. W.: Some Techniques and Uses of 2D-C Habit Classification Software for Snow Particles, J. Atmos. Ocean. Tech., 4, 498-511, https://doi.org/10.1175/15200426(1987)004<0498:stauoc>2.0.co;2, 1987.

Huffman, G. J. and Norman, G. A.: The Supercooled Warm Rain Process and the Specification of Freezing Precipitation, Mon. Weather Rev., 116, 2172-2182, https://doi.org/10.1175/15200493(1988)116<2172:tswrpa>2.0.co;2, 1988.

Hunter, H. E., Dyer, R. M., and Glass, M.: A Two-Dimensional Hydrometeor Machine Classifier Derived from Observed Data, J. Atmos. Ocean. Tech., 1, 28-36, https://doi.org/10.1175/15200426(1984)001<0028:atdhmc>2.0.co;2, 1984.
Jackson, R. C., McFarquhar, G. M., Korolev, A. V., Earle, M. E., Liu, P. S. K., Lawson, R. P., Brooks, S., Wolde, M., Laskin, A., and Freer, M.: The dependence of ice microphysics on aerosol concentration in arctic mixed-phase stratus clouds during ISDAC and M-PACE, J. Geophys. Res.-Atmos., 117, D15207, https://doi.org/10.1029/2012JD017668, 2012

Kajikawa, M., Kikuchi, K., Asuma, Y., Inoue, Y., and Sato, N.: Supercooled drizzle formed by condensation-coalescence in the mid-winter season of the Canadian Arctic, Atmos. Res., 52, 293 301, https://doi.org/10.1016/S0169-8095(99)00035-6, 2000.

Korolev, A.: Reconstruction of the Sizes of Spherical Particles from Their Shadow Images. Part I: Theoretical Considerations, J. Atmos. Ocean. Tech., 24, 376-389, https://doi.org/10.1175/jtech1980.1, 2007.

Korolev, A., Isaac, G., Strapp, J., and Cober, S.: Observation of drizzle at temperatures below $-20^{\circ} \mathrm{C}$, 40th AIAA Aerospace Sciences Meeting \& Exhibit, Reno, NV, USA, https://doi.org/10.2514/6.2002-678, 2002.

Korolev, A., McFarquhar, G., Field, P. R., Franklin, C., Lawson, P., Wang, Z., Williams, E., Abel, S. J., Axisa, D., Borrmann, S., Crosier, J., Fugal, J., Krämer, M., Lohmann, U., Schlenczek, O., Schnaiter, M., and Wendisch, M.: Mixed-Phase Clouds: Progress and Challenges, Meteor. Mon., 58, 5.1-5.50, https://doi.org/10.1175/amsmonographs-d-17-0001.1, 2017.

Korolev, A., Heckman, I., Wolde, M., Ackerman, A. S., Fridlind, A. M., Ladino, L. A., Lawson, R. P., Milbrandt, J., and Williams, E.: A new look at the environmental conditions favorable to secondary ice production, Atmos. Chem. Phys., 20, 13911429, https://doi.org/10.5194/acp-20-1391-2020, 2020.

Korolev, A. V., Kuznetsov, S. V., Makarov, Y. E., and Novikov, V. S.: Evaluation of Measurements of Particle Size and Sample Area from Optical Array Probes, J. Atmos. Ocean. Tech., 8, 514-522, https://doi.org/10.1175/15200426(1991)008<0514:eomops>2.0.co;2, 1991.

Korolev, A. V., Isaac, G. A., Cober, S. G., Strapp, J. W., and Hallett, J.: Microphysical characterization of mixedphase clouds, Q. J. Roy. Meteor. Soc., 129, 39-65, https://doi.org/10.1256/qj.01.204, 2003.

Korolev, A. V., Emery, E. F., Strapp, J. W., Cober, S. G., and Isaac, G. A.: Quantification of the Effects of Shattering on Airborne Ice Particle Measurements, J. Atmos. Ocean. Tech., 30, 2527-2553, https://doi.org/10.1175/jtech-d-13-00115.1, 2013.

Lawson, R. P., O’Connor, D., Zmarzly, P., Weaver, K., Baker, B., Mo, Q., and Jonsson, H.: The 2D-S (Stereo) Probe: Design and Preliminary Tests of a New Airborne, High-Speed, HighResolution Particle Imaging Probe, J. Atmos. Ocean. Tech., 23, 1462-1477, https://doi.org/10.1175/jtech1927.1, 2006.

Lindqvist, H., Muinonen, K., Nousiainen, T., Um, J., McFarquhar, G. M., Haapanala, P., Makkonen, R., and Hakkarainen, H.: Ice-cloud particle habit classification using principal components, J. Geophys. Res.-Atmos., 117, 12, https://doi.org/10.1029/2012jd017573, 2012.

McCoy, D. T., Hartmann, D. L., and Grosvenor, D. P.: Observed Southern Ocean Cloud Properties and Shortwave Reflection. Part I: Calculation of SW Flux from Observed Cloud Properties, J. Climate, 27, 8836-8857, https://doi.org/10.1175/jcli-d14-00287.1, 2014a.

McCoy, D. T., Hartmann, D. L., and Grosvenor, D. P.: Observed Southern Ocean Cloud Properties and Shortwave Reflection. Part 
II: Phase Changes and Low Cloud Feedback, J. Climate, 27, 8858-8868, https://doi.org/10.1175/jcli-d-14-00288.1, 2014 b.

McCoy, D. T., Hartmann, D. L., Zelinka, M. D., Ceppi, P., and Grosvenor, D. P.: Mixed-phase cloud physics and Southern Ocean cloud feedback in climate models, J. Geophys. Res.-Atmos., 120, 9539-9554, https://doi.org/10.1002/2015JD023603, 2015.

McFarquhar, G. M., Um, J., and Jackson, R.: Small Cloud Particle Shapes in Mixed-Phase Clouds, J. Appl. Meteorol. Clim., 52, 1277-1293, https://doi.org/10.1175/jamc-d-12-0114.1, 2013.

McFarquhar, G. M., Finlon, J. A., Stechman, D. M., Wu, W., Jackson, R. C., and Freer, M.: University of Illinois/Oklahoma Optical Array Probe (OAP) Processing Software, Zenodo [code], https://doi.org/10.5281/zenodo.1285969, 2018.

McFarquhar, G. M., Bretherton, C., Marchand, R., Protat, A., DeMott, P. J., Alexander, S. P., Roberts, G. C., Twohy, C. H., Toohey, D., Siems, S., Huang, Y., Wood, R., Rauber, R. M., Lasher-Trapp, S., Jensen, J., Stith, J., Mace, J., Um, J., Järvinen, E., Schnaiter, M., Gettelman, A., Sanchez, K. J., McCluskey, C. S., Russell, L. M., McCoy, I. L., Atlas, R., Bardeen, C. G., Moore, K. A., Hill, T. C. J., Humphries, R. S., Keywood, M. D., Ristovski, Z., Cravigan, L., Schofield, R., Fairall, C., Mallet, M. D., Kreidenweis, S. M., Rainwater, B., D’Alessandro, J., Wang, Y., Wu, W., Saliba, G., Levin, E. J. T., Ding, S., Lang, F., Truong, S. C., Wolff, C., Haggerty, J., Harvey, M. J., Klekociuk, A., and McDonald, A.: Observations of clouds, aerosols, precipitation, and surface radiation over the Southern Ocean: An overview of CAPRICORN, MARCUS, MICRE and SOCRATES, B. Am. Meteorol. Soc., 102, E894-E928, https://doi.org/10.1175/bams-d-20-0132.1, 2020.

Mitchell, J. F. B., Senior, C. A., and Ingram, W. J.: $\mathrm{CO}_{2}$ and climate: a missing feedback?, Nature, 341, 132-134, https://doi.org/10.1038/341132a0, 1989.

Mohrmann, J., Finlon, J., Atlas, R., Lu, J., Hsiao, I., and Wood, R.: University of Washington Ice-Liquid Discriminator single particle phase classifications and $1 \mathrm{~Hz}$ particle size distributions/heterogeneity estimate, Version 1.0, EOL data [data set], https://doi.org/10.26023/PA5W-4DRX-W50A, 2021a.

Mohrmann, J., Finlon, J. A., Lu, J., Hsiao, I., and Atlas, R.: UW Ice Liquid Discriminator (UWILD) cloud particle classification software, Version 1.0, Zenodo [code], https://doi.org/10.5281/zenodo.5197777, 2021b.

Moss, S. J. and Johnson, D. W.: Aircraft measurements to validate and improve numerical model parametrisations of ice to water ratios in clouds, Atmos. Res., 34, 1-25, https://doi.org/10.1016/0169-8095(94)90078-7, 1994.

Murphy, D. M. and Koop, T.: Review of the vapour pressures of ice and supercooled water for atmospheric applications, Q. J. Roy. Meteor. Soc., 131, 1539-1565, https://doi.org/10.1256/qj.04.94, 2005.

Nurzynska, K., Kubo, M., and Muramoto, K.: Texture operator for snow particle classification into snowflake and graupel, Atmos. Res., 118, 121-132, https://doi.org/10.1016/j.atmosres.2012.06.013, 2012.

Nurzynska, K., Kubo, M., and Muramoto, K.: Shape parameters for automatic classification of snow particles into snowflake and graupel, Meteorol. Appl., 20, 257-265, https://doi.org/10.1002/met.299, 2013.
Oraltay, R. G. and Hallett, J.: The Melting Layer: A Laboratory Investigation of Ice Particle Melt and Evaporation near $0{ }^{\circ} \mathrm{C}$, J. Appl. Meteorol., 44, 206-220, https://doi.org/10.1175/jam2194.1, 2005.

O'Shea, S. J., Choularton, T. W., Lloyd, G., Crosier, J., Bower, K. N., Gallagher, M., Abel, S. J., Cotton, R. J., Brown, P. R. A., Fugal, J. P., Schlenczek, O., Borrmann, S., and Pickering, J. C.: Airborne observations of the microphysical structure of two contrasting cirrus clouds, J. Geophys. Res.-Atmos., 121, 13510-13536, https://doi.org/10.1002/2016JD025278, 2016.

Pedregosa, F., Varoquaux, G., Gramfort, A., Michel, V., Thirion, B., Grisel, O., Blondel, M., Prettenhofer, P., Weiss, R., Dubourg, V., Vanderplas, J., Passos, A., Cournapeau, D., Brucher, M., Perrot, M., and Duchesnay, E.: Scikit-learn: Machine Learning in Python, J. Mach. Learn. Res., 12, 2825-2830, 2011.

Praz, C., Roulet, Y.-A., and Berne, A.: Solid hydrometeor classification and riming degree estimation from pictures collected with a Multi-Angle Snowflake Camera, Atmos. Meas. Tech., 10, 13351357, https://doi.org/10.5194/amt-10-1335-2017, 2017.

Praz, C., Ding, S., McFarquhar, G. M., and Berne, A.: A Versatile Method for Ice Particle Habit Classification Using Airborne Imaging Probe Data, J. Geophys. Res.-Atmos., 123, 13472 13495, https://doi.org/10.1029/2018jd029163, 2018.

Radke, L. F., Hobbs, P. V., and Eltgroth, M. W.: Scavenging of Aerosol Particles by Precipitation, J. Appl. Meteorol. Clim., 19, 715-722, https://doi.org/10.1175/15200450(1980)019<0715:soapbp>2.0.co;2, 1980.

Schnaiter, M.: PHIPS-HALO Stereo Imaging Data, Version 1.0, EOL data [data set], https://doi.org/10.5065/D62B8WWF, 2018a.

Schnaiter, M.: PHIPS-HALO Single Particle Data, Version 1.0, EOL data [data set], https://doi.org/10.5065/D6639NKQ, 2018b.

Schnaiter, M., Järvinen, E., Abdelmonem, A., and Leisner, T. PHIPS-HALO: the airborne particle habit imaging and polar scattering probe - Part 2: Characterization and first results, Atmos. Meas. Tech., 11, 341-357, https://doi.org/10.5194/amt-11341-2018, 2018.

Silber, I., Fridlind, A. M., Verlinde, J., Ackerman, A. S., Chen, Y.-S., Bromwich, D. H., Wang, S.-H., Cadeddu, M., and Eloranta, E. W.: Persistent Supercooled Drizzle at Temperatures Below $-25^{\circ} \mathrm{C}$ Observed at McMurdo Station, Antarctica, J. Geophys. Res.-Atmos., 124, 10878-10895, https://doi.org/10.1029/2019JD030882, 2019.

Storelvmo, T., Tan, I., and Korolev, A. V.: Cloud Phase Changes Induced by $\mathrm{CO}_{2}$ Warming-a Powerful yet Poorly Constrained Cloud-Climate Feedback, Current Climate Change Reports, 1, 288-296, https://doi.org/10.1007/s40641-015-0026-2, 2015.

Sun, Z. and Shine, K. P.: Studies of the radiative properties of ice and mixed-phase clouds, Q. J. Roy. Meteor. Soc., 120, 111-137, https://doi.org/10.1002/qj.49712051508, 1994.

Tan, I. and Storelvmo, T.: Sensitivity Study on the Influence of Cloud Microphysical Parameters on Mixed-Phase Cloud Thermodynamic Phase Partitioning in CAM5, J. Atmos. Sci., 73, 709-728, https://doi.org/10.1175/jas-d-15-0152.1, 2016.

Tan, I., Storelvmo, T., and Zelinka, M. D.: Observational constraints on mixed-phase clouds imply higher climate sensitivity, Science, 352, 224-227, https://doi.org/10.1126/science.aad5300, 2016. 
Touloupas, G., Lauber, A., Henneberger, J., Beck, A., and Lucchi, A.: A convolutional neural network for classifying cloud particles recorded by imaging probes, Atmos. Meas. Tech., 13, 2219-2239, https://doi.org/10.5194/amt-13-2219-2020, 2020.

Waitz, F., Schnaiter, M., Leisner, T., and Järvinen, E.: PHIPSHALO: the airborne Particle Habit Imaging and Polar Scattering probe - Part 3: Single-particle phase discrimination and particle size distribution based on the angular-scattering function, Atmos. Meas. Tech., 14, 3049-3070, https://doi.org/10.5194/amt14-3049-2021, 2021.

Wang, Y., McFarquhar, G. M., Rauber, R. M., Zhao, C. F., Wu, W., Finlon, J. A., Stechman, D. M., Stith, J., Jensen, J. B., Schnaiter, M., Jarvinen, E., Waitz, F., Vivekanandan, J., Dixon, M., Rainwater, B., and Toohey, D. W.: Microphysical Properties of Generating Cells Over the Southern Ocean: Results From SOCRATES, J. Geophys. Res.-Atmos., 125, 23, https://doi.org/10.1029/2019jd032237, 2020.

$\mathrm{Wu}, \mathrm{W}$. and McFarquhar, G. M.: NSF/NCAR GV HIAPER fast 2DS particle size distribution (PSD) product data, Version 1.1, UCAR/NCAR [data set], https://doi.org/10.26023/E95A-FKYF7P0R, 2019

Wu, Z. P., Liu, S., Zhao, D. L., Yang, L., Xu, Z. X., Yang, Z. P., Zhou, W., He, H., Huang, M. Y., Liu, D. T., Li, R. J., and Ding, D. P.: Neural Network Classification of Ice-Crystal Images Observed by an Airborne Cloud Imaging Probe, Atmos. Ocean, 58, 303-315, https://doi.org/10.1080/07055900.2020.1843393, 2020.
Xiao, H. X., Zhang, F., He, A. S., Liu, P., Yan, F., Miao, L. J., and Yang, Z. P.: Classification of Ice Crystal Habits Observed From Airborne Cloud Particle Imager by Deep Transfer Learning, Earth and Space Science, 6, 1877-1886, https://doi.org/10.1029/2019ea000636, 2019.

Yang, J., Wang, Z., Heymsfield, A., and Luo, T.: Liquid-Ice Mass Partition in Tropical Maritime Convective Clouds, J. Atmos. Sci., 73, 4959-4978, https://doi.org/10.1175/jas-d-15-0145.1, 2016.

Yuter, S. E., Kingsmill, D. E., Nance, L. B., and Löffler-Mang, M.: Observations of Precipitation Size and Fall Speed Characteristics within Coexisting Rain and Wet Snow, J. Appl. Meteorol. Clim., 45, 1450-1464, https://doi.org/10.1175/jam2406.1, 2006.

Zelinka, M. D., Myers, T. A., McCoy, D. T., Po-Chedley, S., Caldwell, P. M., Ceppi, P., Klein, S. A., and Taylor, K. E.: Causes of Higher Climate Sensitivity in CMIP6 Models, Geophys. Res. Lett., 47, e2019GL085782, https://doi.org/10.1029/2019g1085782, 2020.

Zondlo, M. A., Paige, M. E., Massick, S. M., and Silver, J. A.: Vertical cavity laser hygrometer for the National Science Foundation Gulfstream-V aircraft, J. Geophys. Res.-Atmos., 115, D20309, https://doi.org/10.1029/2010JD014445, 2010. 